\title{
Homogeneity of infinite dimensional anti-Kaehler isoparametric submanifolds
}

\author{
Naoyuki Koike \\ Department of Mathematics, Faculty of Science, Tokyo University of Science, \\ 1-3 Kagurazaka Shinjuku-ku, Tokyo 162-8601 Japan \\ E-mail address: koike@ma.kagu.tus.ac.jp
}

\begin{abstract}
In this paper, we prove that, if a full irreducible infinite dimensional anti-Kaehler isoparametric submanifold of codimension greater than one has $J$-diagonalizable shape operators, then it is homogeneous.
\end{abstract}

Keywords: anti-Kaehler isoparametric submanifold, $J$-principal curvature, J-curvature distribution, regularizability

\section{Introduction}

In 1999, E. Heintze and X. Liu [HL2] proved that all irreducible isoparametric submanifolds of codimension greater than one in the (separable) Hilbert space are homogeneous, which is the infinite dimensional version of the homogeneity theorem for isoparametric submanifolds in a (finite dimensional) Euclidean space by G. Thorbergsson ([Th]). Note that the result of Thorbergsson states that all irreducible isoparametric submanifolds of codimension greater than two in a Euclidean space are homogeneous. In 2002, by using this result of Heintze-Liu, U. Christ [Ch] proved that all irreducible equifocal submanifolds with flat section of codimension greater than one in a simply connected symmetric space of compact type are homogeneous, where we note that, in a simply connected symmetric space of compact type, the notion of an equifocal submanifold coincides with that of an isoparametric submanifold with flat section in the sense of [HLO]. In [Koi1], we introduced the notion of a complex equifocal submanifold in a symmetric space of non-compact type. Here we note that all isoparametric submanifolds with flat section are complex equifocal. In [Koi2], we showed that the study of complex equifocal $C^{\omega}$-submanifolds in the symmetric spaces are reduced to that of anti-Kaehler isoparametric submanifolds in the infinite 
dimensional anti-Kaehler space, where $C^{\omega}$ means the real analyticity. In this paper, we shall investigate an anti-Kaehler isoparametric submanifold with $J$-diagonalizable shape opeartors. According to the discussion in [Koi2], we can show that the study of certain kind of isoparametric submanifolds with flat section in symmetric spaces of non-compact type are reduced to that of anti-Kaehler isoparametric submanifolds with $J$-diagonalizable shape operators in the infinite dimensional anti-Kaehler space, which is called a proper anti-Kaehler isoparametric submanifold in [Koi2]. L. Geatti and C. Gorodski ([GG]) introduced the notion of an isoparametric submanifold with diagonalizable Weingarten operators in a finite dimensional pseudo-Euclidean space. Note that anti-Kaehler isoparametric submanifolds with $J$-diagonalizable shape operators give a subclass of the class of the infinite dimensional version of isoparametric submanifolds with diagonalizable Weingarten operators (see Remark 2.1).

In this paper, we prove the following homogeneity theorem for anti-Kaehler isoparametric $C^{\omega}$-submanifolds with $J$-diagonalizable shape operators in the infinite dimensional anti-Kaehler space.

Theorem A. Let $M$ be a full irreducible anti-Kaehler isoparametric $C^{\omega}$-submanifold with $J$-diagonalizable shape operators of codimension greater than one in the infinite dimensional anti-Kaehler space. Then $M$ is homogeneous.

Remark 1.1. This homogeneity theorem will be useful to prove homogeneity of certain kind of isoparametric submanifolds with flat section in symmetric spaces of non-compact type, which have principal orbits of Hermann actions as homogeneous examples.

\section{Basic notions and facts}

In this section, we shall first recall the notion of an anti-Kaehler isoparametric submanifold in the infinite dimensional anti-Kaehler space introduced in [Koi2]. Let $V$ be an infinite dimensional topological real vector space (or a finite dimensional real vector space), $\widetilde{J}$ a continuous linear operator of $V$ such that $\widetilde{J}^{2}=-$ id and $\langle$,$\rangle a continu-$ ous non-degenerate symmetric bilinear form of $V$ such that $\langle\widetilde{J} X, \widetilde{J} Y\rangle=-\langle X, Y\rangle$ holds for every $X, Y \in V$. Assume that there exists an orthogonal time-space decomposition $V=V_{-} \oplus V_{+}$(i.e., $\left.\langle\rangle\right|_{,V_{-} \times V_{+}}=0,\left.\langle\rangle\right|_{,V_{-} \times V_{-}}$: negative definite, $\left.\langle\rangle\right|_{,V_{+} \times V_{+}}:$positive definite) such that $\widetilde{J} V_{-}=V_{+},\left(V,\langle,\rangle_{V_{ \pm}}\right)$is a separable Hilbert space and that the distance topology associated with $\langle,\rangle_{V_{ \pm}}$coincides with the original topology of $V$, where $\langle,\rangle_{V_{ \pm}}:=-\pi_{V_{-}}^{*}\langle\rangle+,\pi_{V_{+}}^{*}\langle\rangle,\left(\pi_{V_{ \pm}}\right.$: the projection of $V$ onto $\left.V_{ \pm}\right)$. Then we call $(V,\langle\rangle,, \widetilde{J})$ the anti-Keahler space. Let $M$ be a Hilbert manifold modelled on a separable Hilbert space $\left(V^{\prime},\langle,\rangle_{V^{\prime}}\right)$. Let $\langle$,$\rangle be a section of the (0,2)$-tensor bundle $T^{*} M \otimes T^{*} M$ such that $\langle,\rangle_{x}$ is a continuous non-degenerate symmetric bilinear form on $T_{x} M$ for each 
$x \in M$ and $J$ a section of the $(1,1)$-tensor bundle $T^{*} M \otimes T M$ such that $J^{2}=-$ id, $\nabla J=0$ $(\nabla$ : the Levi-Civita connection of $\langle\rangle),, J_{x}$ is a continuous linear operator of $T_{x} M$ for each $x \in M$ and that $\langle J X, J Y\rangle=-\langle X, Y\rangle$ for every $X, Y \in T M$. We call $(M,\langle\rangle, J$, an anti-Keahler Hilbert manifold if, for each $x \in M$, there exist distributions $W_{ \pm}$on some neighborhood $U$ of $x$ satisfying the following condition:

For each $y \in U,\left(W_{ \pm}\right)_{y}$ gives an orthogonal time-space decomposition of $\left(T_{y} M,\langle,\rangle_{y}\right)$ (i.e., $T_{y} M=\left(W_{-}\right)_{y} \oplus\left(W_{+}\right)_{y},\left.\langle,\rangle_{y}\right|_{\left(W_{-}\right)_{y} \times\left(W_{+}\right)_{y}}=0,\left.\langle,\rangle_{y}\right|_{\left(W_{-}\right)_{y} \times\left(W_{-}\right)_{y}}$ : negative definite and $\left.\langle,\rangle_{y}\right|_{\left(W_{+}\right)_{y} \times\left(W_{+}\right)_{y}}$ : positive definite), $\left(T_{y} M,\langle,\rangle_{\left.y,\left(W_{ \pm}\right)_{y}\right)}\right)$ is isometric to $\left(V^{\prime},\langle,\rangle_{V^{\prime}}\right)$ and $J_{y}\left(W_{-}\right)_{y}=\left(W_{+}\right)_{y}$, where $\langle,\rangle_{y,\left(W_{ \pm}\right)_{y}}:=-\pi_{\left(W_{-}\right)_{y}}^{*}\langle,\rangle_{y}+$ $\pi_{\left(W_{+}\right)_{y}}^{*}\langle,\rangle_{y}\left(\pi_{\left(W_{ \pm}\right)_{y}}\right.$ : the projection of $T_{y} M$ onto $\left.\left(W_{ \pm}\right)_{y}\right)$.

Let $f$ be an isometric immersion of an anti-Keahler Hilbert manifold $\left(M,\langle,\rangle_{M}, J\right)$ into an anti-Keahler space $(V,\langle\rangle,, \widetilde{J})$. If $f^{*}\langle\rangle=,\langle,\rangle_{M}$ and if $\widetilde{J} \circ f_{*}=f_{*} \circ J$ holds, then we call $\left(M,\langle,\rangle_{M}, J\right)$ (or $\left.M\right)$ an anti-Kaehler submanifold in $(V,\langle\rangle,, \widetilde{J})$ immersed by $f$. If $M$ is of finite codimension and, for each $v \in T^{\perp} M$, the shape operator $A_{v}$ is a compact operator with respect to $f^{*}\langle,\rangle_{V_{ \pm}}$, then we call $\left(M,\langle,\rangle_{M}, J\right)$ (or $M$ ) an anti-Kaehler Fredholm submanifold. Let $M$ be an anti-Kaehler Fredholm submanifold. Denote by $A$ the shape tensor of $M$. Fix a unit normal vector $v$ of $M$. If there exists $X(\neq 0) \in T M$ with $A_{v} X=a X+b J X$, then we call the complex number $a+b \sqrt{-1}$ a $J$-eigenvalue of $A_{v}$ (or a J-principal curvature of direction $v$ ) and call $X$ a $J$-eigenvector for $a+b \sqrt{-1}$. Also, we call the space of all $J$-eigenvectors for $a+b \sqrt{-1}$ a $J$-eigenspace for $a+b \sqrt{-1}$. The $J$-eigenspaces are orthogonal to one another and they are $J$-invariant, respectively. We call the set of all $J$-eigenvalues of $A_{v}$ the $J$-spectrum of $A_{v}$ and denote it by $\operatorname{Spec}_{J} A_{v}$. Since $M$ is an anti-Kaehler Fredholm submanifold, the set $\operatorname{Spec}_{J} A_{v} \backslash\{0\}$ is described as follows:

$$
\begin{gathered}
\operatorname{Spec}_{J} A_{v} \backslash\{0\}=\left\{\mu_{i} \mid i=1,2, \cdots\right\} \\
\left(\begin{array}{c}
\left|\mu_{i}\right|>\left|\mu_{i+1}\right| \text { or } "\left|\mu_{i}\right|=\left|\mu_{i+1}\right| \& \operatorname{Re} \mu_{i}>\operatorname{Re} \mu_{i+1} " \\
\text { or } "\left|\mu_{i}\right|=\left|\mu_{i+1}\right| \& \operatorname{Re} \mu_{i}=\operatorname{Re} \mu_{i+1} \& \operatorname{Im} \mu_{i}=-\operatorname{Im} \mu_{i+1}>0 "
\end{array}\right) .
\end{gathered}
$$

Also, the $J$-eigenspace for each $J$-eigenvalue of $A_{v}$ other than 0 is of finite dimension. We call the $J$-eigenvalue $\mu_{i}$ the $i$-th $J$-principal curvature of direction $v$. Assume that the normal holonomy group of $M$ is trivial. Fix a parallel normal vector field $\widetilde{v}$ of $M$. Assume that the number (which may be $\infty$ ) of distinct $J$-principal curvatures of direction $\widetilde{v}_{x}$ is independent of the choice of $x \in M$. Then we can define complex-valued functions $\widetilde{\mu}_{i}$ $(i=1,2, \cdots)$ on $M$ by assigning the $i$-th $J$-principal curvature of direction $\widetilde{v}_{x}$ to each $x \in M$. We call this function $\widetilde{\mu}_{i}$ the $i$-th $J$-principal curvature function of direction $\widetilde{v}$. The submanifold $M$ is called an anti-Kaehler isoparametric submanifold if it satisfies the following condition:

The normal holonomy group of $M$ is trivial, and, for each parallel normal vector field 
$\widetilde{v}$ of $M$, the number of distinct $J$-principal curvatures of direction $\widetilde{v}_{x}$ is independent of the choice of $x \in M$, each $J$-principal curvature function of direction $\widetilde{v}$ is constant on $M$ and it has constant multiplicity.

Let $M$ be an anti-Kaehler Fredholm submanifold in $V$. Let $\left\{e_{i}\right\}_{i=1}^{\infty}$ be an orthonormal system of $T_{x} M$. If $\left\{e_{i}\right\}_{i=1}^{\infty} \cup\left\{J e_{i}\right\}_{i=1}^{\infty}$ is an orthonormal base of $T_{x} M$, then we call $\left\{e_{i}\right\}_{i=1}^{\infty}$ (rather than $\left\{e_{i}\right\}_{i=1}^{\infty} \cup\left\{J e_{i}\right\}_{i=1}^{\infty}$ ) a $J$-orthonormal base. If there exists a $J$-orthonormal base consisting of $J$-eigenvectors of $A_{v}$, then we say that $A_{v}$ is diagonalized with respect to a J-orthonormal base (or $A_{v}$ is J-diagonalizable). If, for each $v \in T^{\perp} M$, the shape operator $A_{v}$ is $J$-diagonalizable, then we say that $M$ has $J$-diagonalizable shape operators.

Remark 2.1. If $A_{v}$ is diagonalized with respect to a $J$-orthonormal base, then the complexification $A_{v}^{\mathbf{c}}$ of $A_{v}$ is diagonalized with respect to an orthonormal base. In fact, if $A_{v} X=a X+b J X$, then we have $A_{v}^{\mathbf{c}}(X \pm \sqrt{-1} J X)=(a \mp \sqrt{-1} b)(X \pm \sqrt{-1} J X)$.

Let $M$ be an anti-Kaehler isoparametric submanifold with $J$-diagonalizable shape operators, where we note that such a submanifold was called a proper anti-Kaehler isoparametric submanifold in [Koi2] (in this paper, we do not use this terminology). Then, since the ambient space is flat and the normal holonomy group of $M$ is trivial, it follows from the Ricci equation that the shape operators $A_{v_{1}}$ and $A_{v_{2}}$ commute for arbitrary two normal vector $v_{1}$ and $v_{2}$ of $M$. Hence the shape operators $A_{v}$ 's $\left(v \in T_{x}^{\perp} M\right)$ are simultaneously diagonalized with respect to a $J$-orthonormal base. Let $\left\{E_{0}\right\} \cup\left\{E_{i} \mid i \in I\right\}$ be the family of distributions on $M$ such that, for each $x \in M,\left\{\left(E_{0}\right)_{x}\right\} \cup\left\{\left(E_{i}\right)_{x} \mid i \in I\right\}$ is the set of all common $J$-eigenspaces of $A_{v}$ 's $\left(v \in T_{x}^{\perp} M\right)$, where $\left(E_{0}\right)_{x}=\underset{v \in T_{x}^{\perp} M}{\cap} \operatorname{Ker} A_{v}$. For each $x \in M$, $T_{x} M$ is equal to the closure $\overline{\left(E_{0}\right)_{x} \oplus\left(\oplus_{i \in I}\left(E_{i}\right)_{x}\right)}$ of $\left(E_{0}\right)_{x} \oplus\left(\underset{i \in I}{\oplus}\left(E_{i}\right)_{x}\right)$. We regard $T_{x}^{\perp} M$ $(x \in M)$ as a complex vector space by $\left.J_{x}\right|_{T_{x}^{\perp} M}$ and denote the dual space of the complex vector space $T_{x}^{\perp} M$ by $\left(T_{x}^{\perp} M\right)^{*_{c}}$. Also, denote by $\left(T^{\perp} M\right)^{*_{c}}$ the complex vector bundle over $M$ having $\left(T_{x}^{\perp} M\right)^{*_{\mathrm{c}}}$ as the fibre over $x$. Let $\lambda_{i}(i \in I)$ be the section of $\left(T^{\perp} M\right)^{*_{\mathrm{c}}}$ such that $A_{v}=\operatorname{Re}\left(\lambda_{i}\right)_{x}(v)$ id $+\operatorname{Im}\left(\lambda_{i}\right)_{x}(v) J_{x}$ on $\left(E_{i}\right)_{x}$ for any $x \in M$ and any $v \in T_{x}^{\perp} M$. We call $\lambda_{i}(i \in I) J$-principal curvatures of $M$ and $E_{i}(i \in I) J$-curvature distributions of $M$. The distribution $E_{i}$ is integrable and each leaf of $E_{i}$ is a complex sphere. Each leaf of $E_{i}$ is called a complex curvature sphere. It is shown that there uniquely exists a normal vector field $n_{i}$ of $M$ with $\lambda_{i}(\cdot)=\left\langle n_{i}, \cdot\right\rangle-\sqrt{-1}\left\langle J n_{i}, \cdot\right\rangle$ (see Lemma 5 of [Koi2]). We call $n_{i}(i \in I)$ the $J$-curvature normals of $M$. Note that $n_{i}$ is parallel with respect to the normal connection of $M$. Set $l_{i}^{x}:=\left(\lambda_{i}\right)_{x}^{-1}(1)$. According to (i) of Theorem 2 in [Koi2], the tangential focal set of $M$ at $x$ is equal to $\cup_{i \in I} l_{i}^{x}$. We call each $l_{i}^{x}$ a complex focal hyperplane of $M$ at $x$. Let $\widetilde{v}$ be a parallel normal vector field of $M$. If $\widetilde{v}_{x}$ belongs to at least one $l_{i}$, then it is called a focal normal vector field of $M$. For a focal normal vetor field $\widetilde{v}$, the 
focal map $f_{\widetilde{v}}$ is defined by $f_{\widetilde{v}}(x):=x+\widetilde{v}_{x}(x \in M)$. The image $f_{\widetilde{v}}(M)$ is called a focal submanifold of $M$, which we denote by $F_{\widetilde{v}}$. For each $x \in F_{\widetilde{v}}$, the inverse image $f_{\widetilde{v}}^{-1}(x)$ is called a focal leaf of $M$. Denote by $T_{i}^{x}$ the complex reflection of order 2 with respect to $l_{i}^{x}$ (i.e., the rotation of angle $\pi$ having $l_{i}^{x}$ as the axis), which is an affine transformation of $T_{x}^{\perp} M$. Let $\mathcal{W}_{x}$ be the group generated by $T_{i}^{x}$ 's $(i \in I)$. According to Proposition 3.7 of [Koi3], $\mathcal{W}_{x}$ is discrete. Furthermore, it follows from this fact that $\mathcal{W}_{x}$ is isomorphic to an affine Weyl group. This group $\mathcal{W}_{x}$ is independent of the choice of $x \in M$ (up to group isomorphicness). Hence we simply denote it by $\mathcal{W}$. We call this group the complex Coxeter group associated with $M$. According to Lemma 3.8 of [Koi3], $W$ is decomposable (i.e., it is decomposed into a non-trivial product of two discrete complex reflection groups) if and only if there exist two $J$-invariant linear subspaces $P_{1}(\neq\{0\})$ and $P_{2}(\neq\{0\})$ of $T_{x}^{\perp} M$ such that $T_{x}^{\perp} M=P_{1} \oplus P_{2}$ (orthogonal direct sum), $P_{1} \cup P_{2}$ contains all $J$-curvature normals of $M$ at $x$ and that $P_{i}(i=1,2)$ contains at least one $J$-curvature normal of $M$ at $x$. Also, according to Theorem 1 of [Koi3], $M$ is irreducible if and only if $\mathcal{W}$ is not decomposable.

Next we shall recall the notion of an aks-representation. Let $(N,\langle\rangle, J$,$) be a finite$ dimensional anti-Keahler manifold. If there exists an involutive holomorphic isometry $s_{p}$ of $N$ having $p$ as an isolated fixed point for each $p \in N$, then we call $(N, J,\langle\rangle$, an anti-Keahler symmetric space. Furthermore, if the isometry group of $(N, J,\langle\rangle$,$) is$ semi-simple, then it is said to be semi-simple. Let $G$ be a connected complex Lie group and $K$ a closed complex subgroup of $G$. If there exists an involutive complex automorphism $\rho$ of $G$ such that $G_{\rho}^{0} \subset K \subset G_{\rho}\left(G_{\rho}\right.$ : the group of all fixed points of $\rho$, $G_{\rho}^{0}$ : the identity component of $\left.G_{\rho}\right)$, then we call the pair $(G, K)$ an anti-Keahler symmetric pair. We [Koi4] showed that, for each anti-Kaehler symmetric pair $(G, K)$, the quotient $G / K$ is an anti-Kaehler symmetric space in a natural manner and that, conversely, from each anti-Kaehler symmetric space, an anti-Kaehler symmetric pair arises. Let $\mathfrak{g}$ be a complex Lie algebra and $\tau$ a complex involution of $\mathfrak{g}$. Then we call $(\mathfrak{g}, \tau)$ an anti-Kaehler symmetric Lie algebra. We [Koi4] showed that an anti-Kaehler symmetric Lie algebra arises from an anti-Kaehler symmetric pair and that, conversely, an anti-Kaehler symmetric pair arises from an anti-Kaehler symmetric Lie algebra. Let $(N, J,\langle\rangle$,$) be an irreducible anti-Kaehler symmetric space, G$ the identity component of the holomorphic isometry group of $(N, J,\langle\rangle$,$) and K$ the isotropy group of $G$ at some point $x_{0} \in N$, where the irreducibility implies that $N$ is not decomposed into the non-trivial product of two anti-Kaehler symmetric spaces. Assume that $(N, J,\langle\rangle$,$) does$ not have the pseudo-Euclidean part in its de Rham decomposition. Note that an antiKaehler symmetric space without pseudo-Euclidean part is not necessarily semi-simple (see $[\mathrm{CP}],[\mathrm{W} 1])$. Let $G / K$ be an irreducible anti-Kaehler symmetric space and $(\mathfrak{g}, \tau)$ the anti-Kaehler symmetric Lie algebra associated with $G / K$. Also, set $\mathfrak{p}:=\operatorname{Ker}(\tau+\mathrm{id})$. The space $\operatorname{Ker}(\tau-\mathrm{id})$ is equal to the Lie algebra $\mathfrak{k}$ of $K$ and $\mathfrak{p}$ is identified with $T_{e K}(G / K)$. 
Denote by $\operatorname{Ad}_{G}$ be the adjoint representation of $G$. Define $\left.\operatorname{Ad}_{G}\right|_{\mathfrak{p}}: K \rightarrow \mathrm{GL}(\mathfrak{p})$ by $\left(\left.\operatorname{Ad}_{G}\right|_{\mathfrak{p}}\right)(k):=\left.\operatorname{Ad}_{G}(k)\right|_{\mathfrak{p}}(k \in K)$. We call this representation $\left.\operatorname{Ad}_{G}\right|_{\mathfrak{p}}$ an aks-representation (associated with $G / K$ ). Denote by $\operatorname{ad}_{\mathfrak{g}}$ the adjoint representation of $\mathfrak{g}$. Let $\mathfrak{a}_{s}$ be a maximal split abelian subspace of $\mathfrak{p}$ (see $[\mathrm{R}]$ or $[\mathrm{OS}]$ about the definition of a maximal split abelian subspace) and $\mathfrak{p}=\mathfrak{p}_{0}+\sum_{\alpha \in \triangle_{+}} \mathfrak{p}_{\alpha}$ the root space decomposition with respect to $\mathfrak{a}_{s}$ (i.e., the simultaneously eigenspace decomposition of $\operatorname{ad}_{\mathfrak{g}}(a)^{2}$ 's $\left(a \in \mathfrak{a}_{s}\right)$ ), where the space $\mathfrak{p}_{\alpha}$ is defined by $\mathfrak{p}_{\alpha}:=\left\{X \in \mathfrak{p} \mid \operatorname{ad}_{\mathfrak{g}}(a)^{2}(X)=\alpha(a)^{2} X\right.$ for all $\left.a \in \mathfrak{a}_{s}\right\}\left(\alpha \in \mathfrak{a}_{s}^{*}\right)$ and $\triangle_{+}$is the positive root system of the root system $\triangle:=\left\{\alpha \in \mathfrak{a}_{s}^{*} \mid \mathfrak{p}_{\alpha} \neq\{0\}\right\}$ under some lexicographic ordering of $\mathfrak{a}_{s}^{*}$. Set $\mathfrak{a}:=\mathfrak{p}_{0}\left(\supset \mathfrak{a}_{s}\right), j:=J_{e K}$ and $\langle,\rangle_{0}:=\langle,\rangle_{e K}$. It is shown that $\left.\langle,\rangle_{0}\right|_{\mathfrak{a}_{s} \times \mathfrak{a}_{s}}$ is positive (or negative) definite, $\mathfrak{a}=\mathfrak{a}_{s} \oplus j \mathfrak{a}_{s}$ and $\left.\langle,\rangle_{0}\right|_{\mathfrak{a}_{s} \times j \mathfrak{a}_{s}}=0$. Note that $\mathfrak{p}_{\alpha}=\left\{X \in \mathfrak{p} \mid \operatorname{ad}_{\mathfrak{g}}(a)^{2}(X)=\alpha^{\mathbf{c}}(a)^{2} X\right.$ for all $\left.a \in \mathfrak{a}\right\}$ holds for each $\alpha \in \triangle_{+}$, where $\alpha^{\mathbf{c}}$ is the complexification of $\alpha: \mathfrak{a}_{s} \rightarrow \mathbf{R}$ (which is a complex linear function over $\left.\mathfrak{a}_{s}^{\mathbf{c}}=\mathfrak{a}\right)$ and $\alpha^{\mathbf{c}}(a)^{2} X$ means $\operatorname{Re}\left(\alpha^{\mathbf{c}}(a)^{2}\right) X+\operatorname{Im}\left(\alpha^{\mathbf{c}}(a)^{2}\right) j X$. Let $l_{\alpha}:=\left(\alpha^{\mathbf{c}}\right)^{-1}(0)(\alpha \in \triangle)$ and $D:=\mathfrak{a} \backslash \underset{\alpha \in \triangle_{+}}{\cup} l_{\alpha}$. Elements of $D$ are said to be regular. Take $x \in D$ and let $M$ be the orbit of the aks-representation $\left.\operatorname{Ad}_{G}\right|_{\mathfrak{p}}$ through $x$. From $x \in D, M$ is a principal orbit of this representation. Denote by $A$ the shape tensor of $M$. Take $v \in T_{x}^{\perp} M(=\mathfrak{a})$. Then we have $T_{x} M=\sum_{\alpha \in \triangle_{+}} \mathfrak{p}_{\alpha}$ and $\left.A_{v}\right|_{\mathfrak{p}_{\alpha}}=-\frac{\alpha^{\mathbf{c}}(v)}{\alpha^{\mathbf{c}}(x)} \mathrm{id}\left(\alpha \in \triangle_{+}\right)$. From this fact, we see that $M$ is an anti-Kaehler Fredholm submanifold with $J$-diagonalizable shape operators. Let $\widetilde{v}$ be the parallel normal vector field of $M$ with $\widetilde{v}_{x}=v$. Then we can show that $\left.A_{\widetilde{v}_{\rho(k)(x)}}\right|_{\rho(k)_{* x}\left(\mathfrak{p}_{\alpha}\right)}=-\frac{\alpha^{\mathbf{c}}(v)}{\alpha^{\mathbf{c}}(x)}$ id for any $k \in K$. Hence $M$ is an anti-Kaehler isoparametric submanifold with $J$-diagonalizable shape operators.

\section{Regulalizability of an anti-Kaehler Fredholm submanifold}

In this section, we shall define the regularizability of an anti-Kaehler Fredholm submanifold with $J$-diagonalizable shape operators. Let $\left(M,\langle,\rangle_{M}, J\right)$ be an anti-Kaehler Fredholm submanifold with $J$-diagonalizable shape operators in an infinite dimensional anti-Kaehler space $(V,\langle\rangle,, \widetilde{J})$. Denote by $A$ the shape tensor of $M$. Fix $v \in T^{\perp} M$. Let $\left\{\mu_{i} \mid i=\right.$ $1,2, \cdots\}$ (" $\left|\mu_{i}\right|>\left|\mu_{i+1}\right| "$ or " $\left|\mu_{i}\right|=\left|\mu_{i+1}\right| \& \operatorname{Re} \mu_{i}>\operatorname{Re} \mu_{i+1} "$ or " $\left|\mu_{i}\right|=\left|\mu_{i+1}\right| \& \operatorname{Re} \mu_{i}=$ $\operatorname{Re} \mu_{i+1} \& \operatorname{Im} \mu_{i}=-\operatorname{Im} \mu_{i+1}>0 "$ ") be the set of all $J$-eigenvalues of $A_{v}$ other than zero and $m_{i}$ the multiplicity of $\mu_{i}$. Then we define the regularized trace $\operatorname{Tr}_{r} A_{v}$ of $A_{v}$ by $\operatorname{Tr}_{r} A_{v}:=\sum_{i} m_{i} \mu_{i}$. Also, we define the trace $\operatorname{Tr}_{\text {abs }} A_{v}^{2}$ by $\operatorname{Tr}_{\text {abs }} A_{v}^{2}:=\sum_{i} m_{i}\left|\mu_{i}\right|^{2}$. If there exist $\operatorname{Tr}_{r} A_{v}$ and $\operatorname{Tr}_{\text {abs }} A_{v}^{2}$ for each $v \in T^{\perp} M$, then we say that $M$ is regularizable. It is shown that, if $\mu$ is a $J$-eigenvalue of $A_{v}$ with multiplicity $m$, then so is also the conjugate $\bar{\mu}$ of $\mu$. Hence we have $\operatorname{Tr}_{r} A_{v} \in \mathbb{R}$. Define $H_{x} \in T_{x}^{\perp} M$ by $\left\langle H_{x}, v\right\rangle=\operatorname{Tr}_{r} A_{v}\left(\forall v \in T_{x}^{\perp} M\right)$. We call the normal vector field $H\left(: x \mapsto H_{x}\right)$ of $M$ the regularized mean curvature vector of $M$. 


\section{Proof of Theorem A}

In this section, we shall prove Theorem A. For its purpose, we shall prepare some lemmas (and theorems). First we shall recall the generalized Chow's theorem, which was proved in [HL2]. Let $N$ be a (connected) Hilbert manifold and $\mathcal{D}$ a set of local (smooth) vector fields which are defined over open sets of $N$. If two points $x$ and $y$ of $N$ can be connected by a piecwise smooth curve each of whose smooth segments is an integral curve of a local smooth vector field belonging to $\mathcal{D}$, then we say that $x$ and $y$ are $\mathcal{D}$-equivalent and we denote this fact by $x \underset{\mathcal{D}}{\sim} y$. Let $\Omega_{\mathcal{D}}(x):=\{y \in N \mid y \underset{\mathcal{D}}{\sim} x\}$. The set $\Omega_{\mathcal{D}}(x)$ is called the set of reachable points of $\mathcal{D}$ starting from $x$. Let $\mathcal{D}^{*}$ be the minimal set consisting of local smooth vector fields on open sets of $N$ which satisfies the following condition:

$\mathcal{D} \subset \mathcal{D}^{*}$ and $\mathcal{D}^{*}$ contains the zero vector field and, for any $X, Y \in \mathcal{D}^{*}$ and any $a, b \in \mathbf{R}$, $a X+b Y$ and $[X, Y]$ (which are defined on the intersection of the domains of $X$ and $Y$ ) also belong to $\mathcal{D}^{*}$.

For each $x \in N$, set $\mathcal{D}^{*}(x):=\left\{X_{x} \mid X \in \mathcal{D}^{*}\right.$ s.t. $\left.x \in \operatorname{Dom}(X)\right\}$. Then the following generalized Chow's theorem holds.

Theorem 4.1([HL2]) If $\overline{\mathcal{D}^{*}(x)}=T_{x} N$ for each $x \in N, \overline{\Omega_{\mathcal{D}}(x)}=N$ holds for each $x \in N$, where $\overline{(\cdot)}$ implies the closure of $(\cdot)$.

Let $M$ be as in the statement of Theorem A. Denote by $\left(\langle,\rangle_{M}, J\right)$ and $A$ the antiKaehler structure and the shape tensor of $M$, respectively. For simplicity, we denote $\langle,\rangle_{M}$ by $\langle$,$\rangle . Let \left\{E_{0}\right\} \cup\left\{E_{i} \mid i \in I\right\}$ the set of all $J$-curvature distributions of $M$, where $E_{0}$ is defined by $\left(E_{0}\right)_{x}:=\underset{v \in T_{x}^{\perp} M}{\cap} \operatorname{Ker} A_{v}(x \in M)$. Also, let $\lambda_{i}$ and $n_{i}$ be the $J$-principal curvature and the $J$-curvature normal corresponding to $E_{i}$, respectively. Denote by $l_{i}^{x}$ the complex focal hyperplane $\left(\lambda_{i}\right)_{x}^{-1}(1)$ of $M$ at $x$. Also set $\left(l_{i}^{x}\right)^{\prime}:=\left(\lambda_{i}\right)_{x}^{-1}(0)$. Fix $x_{0} \in M$. For simplicity, set $l_{i}:=l_{i}^{x_{0}}$ and $l_{i}^{\prime}:=\left(l_{i}^{x_{0}}\right)^{\prime}$. Let $Q\left(x_{0}\right)$ be the set of all points of $M$ connected with $x_{0}$ by a piecewise smooth curve in $M$ each of whose smooth segments is contained in some complex curvature sphere (which may depend on the smooth segment). By using the above generalized Chow's theorem, we shall show the following result.

Proposition 4.2. The set $Q\left(x_{0}\right)$ is dense in $M$.

Proof. Let $\mathcal{D}_{E}$ be the set of all local (smooth) tangent vector fields on open sets of $M$ which is tangent to some $E_{i}(i \neq 0)$ at each point of the domain. Define $\Omega_{\mathcal{D}_{E}}\left(x_{0}\right), \mathcal{D}_{E}^{*}$ and $\mathcal{D}_{E}^{*}\left(x_{0}\right)$ as above. By imitating the proof of Proposition 5.8 of [HL2], it is shown that $\overline{\mathcal{D}_{E}^{*}(x)}=T_{x} M$ for each $x \in M$. Hence, $\overline{\Omega_{\mathcal{D}_{E}}\left(x_{0}\right)}=M$ follows from Theorem 4.1. It is clear that $\Omega_{\mathcal{D}_{E}}\left(x_{0}\right)=Q\left(x_{0}\right)$. Therefore we obtain $\overline{Q\left(x_{0}\right)}=M$ q.e.d. 
For each complex affine subspace $P$ of $T_{x_{0}}^{\perp} M$, define $I_{P}$ by

$$
I_{P}:= \begin{cases}\left\{i \in I \mid\left(n_{i}\right)_{x_{0}} \in P\right\} & (0 \notin P) \\ \left\{i \in I \mid\left(n_{i}\right)_{x_{0}} \in P\right\} \cup\{0\} & (0 \in P) .\end{cases}
$$

Define a distribution $D_{P}$ on $M$ by $D_{P}:=\underset{i \in I_{P}}{\bigoplus} E_{i}$.

Lemma 4.3. The following statements hold:

(i) $M$ is regularizable.

(ii) If $0 \notin P$, then $I_{P}$ is finite and $\left(\underset{i \in I_{P}}{\cap} l_{i}\right) \backslash\left(\underset{i \in I \backslash I_{P}}{\cup} l_{i}\right) \neq \emptyset$.

(iii) If $0 \in P$, then $I_{P}$ is infinite or $I_{P}=\{0\}$ and $\left(\underset{i \in I_{P} \backslash\{0\}}{\cap} l_{i}^{\prime}\right) \backslash\left(\underset{i \in I \backslash I_{P}}{\cup} l_{i}^{\prime}\right) \neq \emptyset$, where $\underset{i \in I_{P} \backslash\{0\}}{\cap} l_{i}^{\prime}$ means $T_{x_{0}}^{\perp} M$ when $I_{P}=\{0\}$.

Proof. From the discreteness of the complex Coxeter group associated with $M$, we can show that $B:=\left\{\left(n_{i}\right)_{x_{0}} \mid i \in I\right\}$ is described as $B=\left\{\frac{1}{1+a_{i j}}\left(n_{i}\right)_{x_{0}} \mid i \in I_{0}, j \in \mathbb{Z}\right\}$ in terms of some finite subset $I_{0}$ of $I$ and some set $\left\{a_{i} \mid i \in I_{0}\right\}$ of complex numbers. From this fact, the statements in this lemma follow. q.e.d.

Assume that $0 \notin P$. Take $v \in\left(\underset{i \in I_{P}}{\cap} l_{i}\right) \backslash\left(\underset{i \in I \backslash I_{P}}{\cup} l_{i}\right)$. Let $\widetilde{v}$ be a parallel normal vector field on $M$ with $\widetilde{v}_{x_{0}}=v$. This normal vector field $\widetilde{v}$ is a focal normal vector field of $M$. Let $f_{\widetilde{v}}$ be the focal map (i.e., the end point map) for $\widetilde{v}$ and $F_{\widetilde{v}}$ the focal submanifold for $\widetilde{v}$ (i.e., $F_{\widetilde{v}}=f_{\widetilde{v}}(M)$ ). Also, let $L_{x}^{D_{P}}$ be the leaf of $D_{P}$ through $x \in M$. Note that $L_{x}^{D_{P}}=f_{\widetilde{v}}^{-1}\left(f_{\widetilde{v}}(x)\right)$. Now we shall show the following homogeneous slice theorem for $M$.

Theorem 4.4. If $0 \notin P$, then the leaf $L_{x}^{D_{P}}\left(\subset T_{f_{\tilde{v}}(x)}^{\perp} F_{\widetilde{v}}\right)$ is a principal orbit of the direct sum representation of some aks-representations and a trivial representation.

We shall recall the notion of an anti-Kaehler holonomy system introduced in [Koi4] to prove this theorem. Let $(W, J,\langle\rangle$,$) be a (finite dimensional) anti-Kaehler space and$ $R\left(\in W^{*} \otimes W^{*} \otimes W^{*} \otimes W\right)$ a curvature-like tensor. Also, let $S O_{A K}(W)$ be the identity component of the group $\left\{B \in G L(W) \mid B^{*}\langle\rangle=,\langle\rangle,,[B, J]=0\right\}$ and $G$ a connected complex Lie subgroup of $S O_{A K}(W)$. We call the triple $((W, J,\langle\rangle), R, G$,$) an anti-Kaehler$ holonomy system if the following two conditions hold:

(i) $J \circ R\left(w_{1}, w_{2}\right)=R\left(J w_{1}, w_{2}\right)=R\left(w_{1}, w_{2}\right) \circ J$ for all $w_{1}, w_{2} \in W$,

(ii) $R\left(w_{1}, w_{2}\right) \in \operatorname{Lie} G$ for all $w_{1}, w_{2} \in W$.

Furthermore, if the following condition (iii) holds, then we say that the triple is symmetric:

(iii) $R\left(g w_{1}, g w_{2}\right) g w_{3}=g R\left(w_{1}, w_{2}\right) w_{3}$ for all $w_{i} \in W(i=1,2,3)$ and all $g \in G$.

Also, if $G$ is weakly irreducible, then we say that the triple is weakly irreducible, where the weakly irreduciblity of $G$ implies that there exists no $G$-invariant non-degenerate 
subspace $W^{\prime}$ of $W$ with $W^{\prime} \neq\{0\}$ and $W^{\prime} \neq W$ (where the non-degeneracy of $W^{\prime}$ impies that $\left.\langle\rangle\right|_{,W^{\prime} \times W^{\prime}}$ is non-degenerate). We [Koi4] proved the following fact for a weakly irreducible symmetric anti-Kaehler holonomy system.

Lemma 4.4.1. For a weakly irreducible symmetric anti-Kaehler holonomy system $((W, J,\langle\rangle), R, G$,$) with R \neq 0$, the $G$-action on $W$ is equivalent to an aks-representation.

By using this lemma, we prove Theorem 4.4.

Proof of Theorem 4.4. Set $x^{\prime}:=f_{\widetilde{v}}(x)$. Denote by $\Psi\left(x^{\prime}\right)$ the normal holonomy group of $F_{\widetilde{v}}$ at $x^{\prime}$ and $\Psi^{0}\left(x^{\prime}\right)$ the identity component of $\Psi\left(x^{\prime}\right)$. Since $\operatorname{dim} T_{x^{\prime}}^{\perp} F_{\widetilde{v}}<\infty, \Psi^{0}\left(x^{\prime}\right)$ is a Lie subgroup of $S O_{A K}\left(T_{x^{\prime}}^{\perp} F_{\widetilde{v}}\right)$. It is clear that $\Psi^{0}\left(x^{\prime}\right)$ is not trivial. For simplicity, set $W:=T_{x^{\prime}}^{\perp} F_{\widetilde{v}}$. Let $W=W_{0} \oplus W_{1} \oplus \cdots \oplus W_{k}$ be the weakly irreducible decomposition of the $\Psi^{0}\left(x^{\prime}\right)$-module $W$, where $\left.\Psi^{0}\left(x^{\prime}\right)\right|_{W_{0}}=\left\{\operatorname{id}_{W_{0}}\right\}$ and $W_{i}(i=1, \cdots, k)$ are (nontrivial) weakly irreducible $\Psi^{0}\left(x^{\prime}\right)$-submodules of $W$. For simplicity, set $\Psi_{i}^{0}\left(x^{\prime}\right):=\left.\Psi^{0}\left(x^{\prime}\right)\right|_{W_{i}}$ $(i=1, \cdots, k)$. Denote by $\widehat{A}$ the shape tensor of $F_{\widetilde{v}}$ and $R^{\perp}$ the curvature tensor of the normal connection of $F_{\widetilde{v}}$. Also, denote by $\mathcal{L}^{2}$ the space of Hilbert-Schmidt operators of the Hilbert space $\left(T_{x^{\prime}} F_{\widetilde{v}},\left.\langle,\rangle_{V_{ \pm}}\right|_{x_{x^{\prime}} F_{\widetilde{v}} \times T_{x^{\prime}} F_{\widetilde{v}}}\right)$ and $\langle,\rangle_{\mathcal{L}^{2}}$ the Hilbert-Schmidt inner product of $\mathcal{L}^{2}$. Define $\mathcal{R}_{i}^{\perp} \in W_{i}^{*} \otimes W_{i}^{*} \otimes W_{i}^{*} \otimes W_{i}$ by

$$
\left\langle\mathcal{R}_{i}^{\perp}\left(w_{1}, w_{2}\right) w_{3}, w_{4}\right\rangle:=-\frac{1}{2}\left\langle\left[\widehat{A}_{w_{1}}, \widehat{A}_{w_{2}}\right],\left[\widehat{A}_{w_{3}}, \widehat{A}_{w_{4}}\right]\right\rangle_{\mathcal{L}^{2}} \quad\left(w_{1}, \cdots w_{4} \in W_{i}\right) .
$$

Here we note that $\widehat{A}_{w_{j}}$ 's $(j=1, \cdots, 4)$ are Hilbert-Schmidt operators because $M$ (hence $\left.F_{\widetilde{v}}\right)$ is a regulalizable anti-Kaehler Fredholm submanifold with $J$-diagonalizable shape operators. From the Ricci equation, $\left[\widehat{A}_{w_{j}}, J\right]=0$ and $R^{\perp}(J X, J Y)=-R^{\perp}(X, Y)(X, Y \in$ $T_{x^{\prime}} F_{\widetilde{v}}$, we can show

$$
\left\langle\mathcal{R}_{i}^{\perp}\left(w_{1}, w_{2}\right) w_{3}, w_{4}\right\rangle=2 \sum_{j \in \mathbb{N}}\left\langle R^{\perp}\left(\widehat{A}_{w_{1}} e_{j}, \widehat{A}_{w_{2}} e_{j}\right) w_{3}, w_{4}\right\rangle_{V_{ \pm}} \quad\left(w_{1}, \cdots w_{4} \in W_{i}\right),
$$

where $\left\{e_{j}\right\}_{j=1}^{\infty}$ is a $J$-orthonormal base of $T_{x^{\prime}} F_{\widetilde{v}}$. By using this relation, we can show that $\left(W_{i}, \mathcal{R}_{i}^{\perp}, \Psi_{i}^{0}\left(x^{\prime}\right)\right)$ is a weakly irreducible symmetric anti-Kaehler holonomy system. Also, from $\left.R^{\perp}\right|_{T_{x^{\prime}} F_{\widetilde{v}} \times T_{x^{\prime}} F_{\widetilde{v}} \times W_{i}} \neq 0$, we can show $\mathcal{R}_{i}^{\perp} \neq 0$. Hence it follows from Lemma 4.4.1 that the $\Psi_{i}^{0}\left(x^{\prime}\right)$-action on $W_{i}$ is equivalent to an aks-representation. Also, the $\Psi_{0}^{0}\left(x^{\prime}\right)$ action on $W_{0}$ is trivial. Therefore, since $L_{x}^{D_{P}}$ is a principal orbit of $\Psi^{0}\left(x^{\prime}\right)$-action, the statement of Theorem 4.4 follows.

q.e.d.

Set $\left(W_{P}\right)_{x}:=x+\left(D_{P}\right)_{x} \oplus \operatorname{Span}_{\mathbf{C}}\left\{\left(n_{i}\right)_{x} \mid i \in I_{P} \backslash\{0\}\right\}(x \in M)$. Let $\gamma:[0,1] \rightarrow M$ be a piecewise smooth curve. In the sequel, we assume that the domains of all piecewise smooth curves are equal to $[0,1]$. If $\dot{\gamma}(t) \perp\left(D_{P}\right)_{\gamma(t)}$ for each $t \in[0,1]$, then $\gamma$ is said to be horizontal with respect to $D_{P}$ (or $D_{P}$-horizontal). Let $\beta_{i}(i=1,2)$ be curves in $M$. If $L_{\beta_{1}(t)}^{D_{P}}=L_{\beta_{2}(t)}^{D_{P}}$ for each $t \in[0,1]$, then $\beta_{1}$ and $\beta_{2}$ are said to be parallel with respect to $D_{P}$. By imitating the proof of Proposition 1.1 in [HL2], we can show the following fact. 
Lemma 4.5. For each $D_{P}$-horizontal curve $\gamma$, there exists an one-parameter family $\left\{h_{\gamma, t}^{D_{P}} \mid 0 \leq t \leq 1\right\}$ of holomorphic isometries $h_{\gamma, t}^{D_{P}}:\left(W_{P}\right)_{\gamma(0)} \rightarrow\left(W_{P}\right)_{\gamma(t)}$ satisfying the following conditions:

(i) $h_{\gamma, t}^{D_{P}}\left(L_{\gamma(0)}^{D_{P}}\right)=L_{\gamma(t)}^{D_{P}}(0 \leq t \leq 1)$,

(ii) for any $x \in L_{\gamma(0)}^{D_{P}}, t \mapsto h_{\gamma, t}^{D_{P}}(x)$ is a $D_{P}$-horizontal curve parallel to $\gamma$,

(iii) for any $x \in L_{\gamma(0)}^{D_{P}}$ and any $i \in I_{P},\left(h_{\gamma, t}^{D_{P}}\right)_{* x}\left(\left(E_{i}\right)_{x}\right)=\left(E_{i}\right)_{h_{\gamma, t}^{D_{P}}(x)}$.

Proof. First we consider the case of $0 \notin P$. Take $v \in \underset{i \in I_{P}}{\cap} l_{i} \backslash\left(\underset{i \in I \backslash I_{P}}{\cup} l_{i}\right)$. Let $\widetilde{v}$ be the parallel normal vector field of $M$ with $\widetilde{v}_{x_{0}}=v$. Let $\bar{\gamma}:=f_{\widetilde{v}} \circ \gamma$. Define a map $h_{t}:\left(W_{P}\right)_{\gamma(0)} \rightarrow V$ by $h_{t}(x):=\bar{\gamma}(t)+\bar{\tau} \frac{\perp}{\left.\bar{\gamma}\right|_{[0, t]}}(\overrightarrow{\bar{\gamma}(0) x})\left(x \in\left(W_{P}\right)_{\gamma(0)}\right)$ (see Figure 1), where $\bar{\tau} \frac{\perp}{\gamma}$ is the parallel translation along $\bar{\gamma}$ with respect to the normal connection of $F_{\widetilde{v}}$. Then it is shown that $\left\{h_{t} \mid 0 \leq t \leq 1\right\}$ is the desired one-parameter family. Next we consider the case of $0 \in P$. Take $v \in \underset{i \in I_{P} \backslash\{0\}}{\cap} l_{i}^{\prime} \backslash\left(\underset{i \in I \backslash I_{P}}{\cup} l_{i}^{\prime}\right)$. Let $\widetilde{v}$ be the parallel normal vector field of $M$ with $\widetilde{v}_{x_{0}}=v$. We define a map $\nu: M \rightarrow S^{\infty}(1)$ by $\nu(x):=\widetilde{v}_{x}(x \in M)$, where $S^{\infty}(1)$ is the unit hypersphere of $V$ centered 0 . Then we have $\nu_{* x}=-A_{\widetilde{v}_{x}}(x \in M)$. If $i \in I_{P}$, then we have $\nu_{* x}\left(\left(E_{i}\right)_{x}\right)=\left\{-\left\langle\left(n_{i}\right)_{x}, \widetilde{v}_{x}\right\rangle X \mid X \in\left(E_{i}\right)_{x}\right\}=\{0\}$ and, if $i \notin I_{P}$, then we have $\nu_{* x}\left(\left(E_{i}\right)_{x}\right)=\left\{-\left\langle\left(n_{i}\right)_{x}, \widetilde{v}_{x}\right\rangle X \mid X \in\left(E_{i}\right)_{x}\right\}=\left(E_{i}\right)_{x}$. Hence we have $\operatorname{Ker} \nu_{* x}=\left(D_{P}\right)_{x}$. Therefore $D_{P}$ is integrable and it gives a foliation on $M$. Denote by $\mathfrak{F}_{P}$ this foliation and $D_{P}^{\perp}$ the orthogonal complementary distribution of $\mathfrak{F}_{P}$. Let $U$ be a neighborhood of $\gamma(0)$ in $L_{\gamma(0)}^{D_{P}}$ such that there exists a family $\left\{\psi_{t}: U \rightarrow U_{t} \mid 0 \leq t \leq 1\right\}$ of diffeomophisms such that, for any $x \in U$, the curve $\gamma_{x}\left(\underset{\text { def }}{\Leftrightarrow} \gamma_{x}(t):=\psi_{t}(x)\right)$ is a $D_{P}$-horizontal curve, where $U_{t}$ is a neighborhood of $\gamma(t)$ in $L_{\gamma(t)}^{D_{P}}$. Note that such a family of diffeomorphisms is called an element of holonomy along $\gamma$ (with respect to $\mathcal{F}_{P}$ and $D_{P}^{\perp}$ ) in [BH]. Let $\triangle$ be a fundamental domain containing $x_{0}$ of the complex Coxeter group of $M$ at $x_{0}$. Denote by $\triangle_{x}$ a domain of $T_{x}^{\perp} M$ given by parallel translating $\triangle$ with respect to the normal connection of $M$. Set $\widetilde{U}:=\cup_{x \in U}\left(\operatorname{Span}_{\mathbf{C}}\left\{\left(n_{i}\right)_{x} \mid i \in I_{P} \backslash\{0\}\right\} \cap \triangle_{x}\right)$, which is an open subset of the affine subspace $\left(W_{P}\right)_{\gamma(0)}$. Define a map $h_{t}: \widetilde{U} \rightarrow\left(W_{P}\right)_{\gamma(t)}(0 \leq t \leq 1)$ by $h_{t}(x+w)=\gamma_{x}(t)+\tau_{\left.\gamma_{x}\right|_{[0, t]}}^{\perp}(w)\left(x \in U, w \in \operatorname{Span}\left\{\left(n_{i}\right)_{x} \mid i \in I_{P} \backslash\{0\}\right\} \cap \triangle_{x}\right)$ (see Figure 2 ). By imitating the proof of Lemma 1.2 in [HL2], it is shown that $h_{t}$ is a holomorphic isometry into $\left(W_{P}\right)_{\gamma(t)}$. Hence $h_{t}$ extends to a holomorphic isometry of $\left(W_{P}\right)_{\gamma(0)}$ onto $\left(W_{P}\right)_{\gamma(t)}$. Denote by $\widetilde{h}_{t}$ this holomorphic extension. It is shown that $\widetilde{h}_{t}$ 's gives the desired one-parameter family by imitating the discussion in Step 3 of the proof of Proposition 1.1 in [HL2].

q.e.d. 


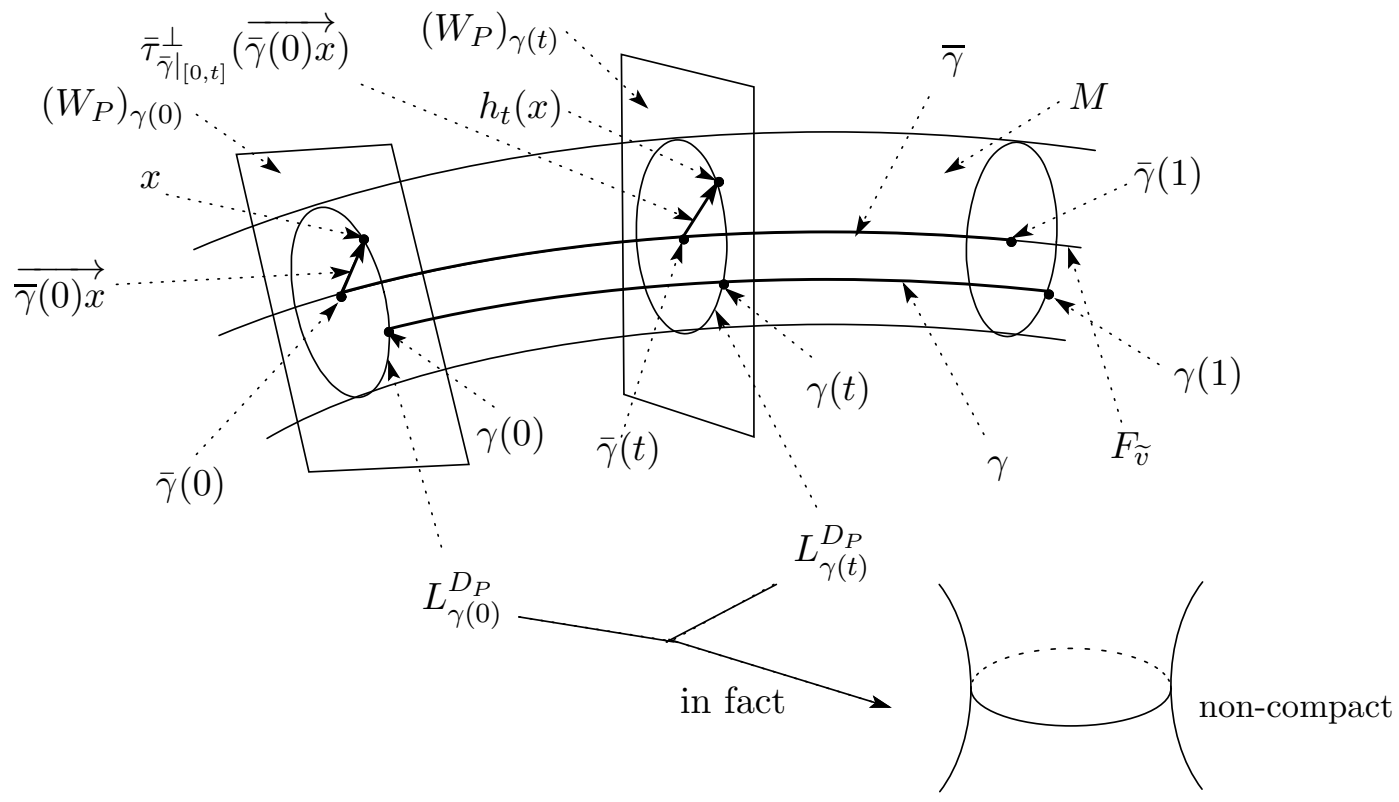

Figure 1.

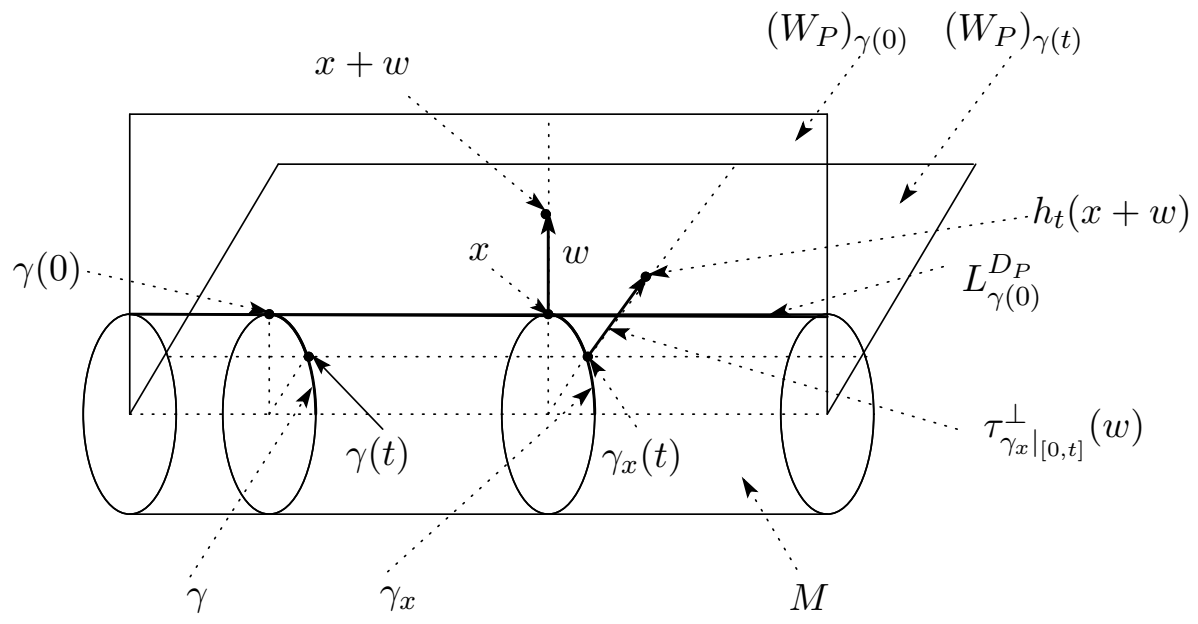

Figure 2. 
Fix $x_{0} \in M$ and $i_{0} \in I \cup\{0\}$. Take a complex affine subspace $P_{i_{0}}$ of $T_{x_{0}}^{\perp} M$ with $I_{P_{i_{0}}}=\left\{i_{0}\right\}$. Note that $D_{P_{i_{0}}}$ is equal to $E_{i_{0}}$. Denote by $\Phi_{i_{0}}\left(x_{0}\right)$ the group of holomorphic isometries of $\left(W_{P_{i_{0}}}\right)_{x_{0}}$ generated by $\left\{h_{\gamma, 1}^{E_{i_{0}}} \mid \gamma: E_{i_{0}}\right.$ - horizontal curve s.t. $\left.\gamma(0), \gamma(1) \in L_{x_{0}}^{E_{i_{0}}}\right\}$, where $L_{x_{0}}^{E_{i_{0}}}$ is the integral manifold of $E_{i_{0}}$ through $x_{0}$. Also, denote by $\Phi_{i_{0}}^{0}\left(x_{0}\right)$ the identity component of $\Phi_{i_{0}}\left(x_{0}\right)$ and $\Phi_{i_{0}}^{0}\left(x_{0}\right)_{x_{0}}$ the isotropy subgroup of $\Phi_{i_{0}}^{0}\left(x_{0}\right)$ at $x_{0}$. Define a $\operatorname{Ad}_{\Phi_{i_{0}}^{0}\left(x_{0}\right)}\left(\Phi_{i_{0}}^{0}\left(x_{0}\right)\right)$-invariant non-degenerate inner product $\langle$,$\rangle of the Lie algebra$ Lie $\Phi_{i_{0}}^{0}\left(x_{0}\right)$ of $\Phi_{i_{0}}^{0}\left(x_{0}\right)$ by

$$
\langle X, Y\rangle:=B(X, Y)+\operatorname{Tr}(X \circ Y) \quad\left(X, Y \in \operatorname{Lie} \Phi_{i_{0}}^{0}\left(x_{0}\right)\right)
$$

where $B$ is the Killing form of Lie $\Phi_{i_{0}}^{0}\left(x_{0}\right)$ and $X \circ Y$ implies the composition of $X$ and $Y$ regarded as linear transformations of $\left(W_{P_{i_{0}}}\right)_{x_{0}}$. Take $X \in \operatorname{Lie} \Phi_{i_{0}}^{0}\left(x_{0}\right) \ominus \operatorname{Lie} \Phi_{i_{0}}^{0}\left(x_{0}\right)_{x_{0}}$. Set $g(t):=\exp t X$ and $\gamma(t):=g(t) x_{0}$, where exp is the exponential map of $\Phi_{i_{0}}^{0}\left(x_{0}\right)$. It is clear that $\gamma$ is an $E_{i}$-horizontal curve for each $i \in I$ with $i \neq i_{0}$. Let $F_{\gamma}$ be the holomorphic isometry of $V$ satisfying $F_{\gamma}(\gamma(0))=\gamma(1)$ and

$$
\left(F_{\gamma}\right)_{* \gamma(0)}= \begin{cases}g(1)_{* \gamma(0)} & \text { on }\left(E_{i_{0}}\right)_{\gamma(0)} \\ \left(h_{\gamma, 1}^{E_{i}}\right)_{* \gamma(0)} & \text { on }\left(E_{i}\right)_{\gamma(0)}\left(i \in(I \cup\{0\}) \backslash\left\{i_{0}\right\}\right) \\ \tau_{\gamma}^{\perp} & \text { on } T_{\gamma(0)}^{\perp} M .\end{cases}
$$

In similar to Theorem 4.1 of [HL2], we have the following fact.

Proposition 4.6. The holomorphic isometry $F_{\gamma}$ preserves $M$ invariantly (i.e., $F_{\gamma}(M)=$ $M)$. Furthermore, it preserves $E_{i}(i \in I \cup\{0\})$ invariantly (i.e., $\left.F_{\gamma *}\left(E_{i}\right)=E_{i}\right)$.

To show this proposition, we prepare some lemmas. By imitating the proof (P163 166) of Proposition 3.1 in [HL2], we can show the following fact.

Lemma 4.6.1. Let $N$ and $\widehat{N}$ be full irreducible anti-Kaehler isoparametric submanifolds with $J$-diagonalizable shape operators in an infinite dimensional anti-Kaehler space. If $\operatorname{codim}_{\mathbf{c}} N=\operatorname{codim}_{\mathbf{c}} \widehat{N} \geq 2, N \cap \widehat{N} \neq \emptyset$ and, for some $x_{0} \in N \cap \widehat{N}, T_{x_{0}} N=T_{x_{0}} \widehat{N}$ and if there exists a complex affine line $l_{0}$ of $T_{x_{0}}^{\perp} N\left(=T_{x_{0}}^{\perp} \widehat{N}\right)$ such that $L_{x_{0}}^{D_{l}}=L_{x_{0}}^{\widehat{D}_{l}}$ for any complex affine line $l$ of $T_{x_{0}}^{\perp} N$ with $l \neq l_{0}$, then $N=\widehat{N}$ holds, where $D_{l}$ (resp. $\widehat{D}_{l}$ ) is the integrable distribution on $N$ (resp. $\widehat{N}$ ) defined for $l$ in similar to $D_{P}$.

Proof. Let $\left\{\lambda_{i} \mid i \in I\right\}$ (resp. $\left\{\widehat{\lambda}_{i} \mid i \in \widehat{I}\right\}$ ) be the set of all $J$-principal curvatures of $N$ (resp. $\widehat{N}), \mathbf{n}_{i}$ (resp. $\widehat{\mathbf{n}}_{i}$ ) the $J$-curvature normal corresponding to $\lambda_{i}\left(\right.$ resp. $\left.\widehat{\lambda}_{i}\right)$ and $E_{i}$ (resp. $\left.\widehat{E}_{i}\right)$ the $J$-curvature distribution corresponding to $\lambda_{i}$ (resp. $\widehat{\lambda}_{i}$ ). Denote by $A$ (resp. $\widehat{A}$ ) the shape tensor of $N$ (resp. $\widehat{N}$ ). Let $E_{0}$ be the $J$-curvature distribution 
on $N$ with $\left(E_{0}\right)_{x}:=\bigcap_{v \in T_{x}^{\perp} N} \operatorname{Ker} A_{v}(x \in N)$ and $\widehat{E}_{0}$ the $J$-curvature distribution on $\widehat{N}$ with $\left(\widehat{E}_{0}\right)_{x}:=\bigcap_{v \in T_{x}^{\perp} \widehat{N}} \operatorname{Ker} \widehat{A}_{v}(x \in \widehat{N})$. For each $x \in N$ (resp. $\left.\hat{x} \in \widehat{N}\right)$, let $Q_{0}(x)$ (resp. $\left.\widehat{Q}_{0}(\hat{x})\right)$ be the set of all points of $N$ (resp. $\left.\widehat{N}\right)$ connected with $x$ (resp. $\hat{x}$ ) by a piecewise smooth curve in $N$ (resp. $\widehat{N}$ ) each of whose smooth segments is contained in some complex curvature sphere in $N$ (resp. $\widehat{N}$ ) or some integral manifold of $E_{0}$ (resp. $\widehat{E}_{0}$ ). Take any $x \in Q_{0}\left(x_{0}\right)$. There exists a sequence $\left\{x_{0}, x_{1}, \cdots, x_{k}(=x)\right\}$ such that, for each $j \in\{1, \cdots, k\}, x_{j} \in\left(\cup_{i \in I} L_{x_{j-1}}^{E_{i}}\right) \cup L_{x_{j-1}}^{E_{0}}$ holds. Assume that there exists $j_{0} \in\{1, \cdots, k\}$ such that $x_{j_{0}} \in L_{x_{j_{0}-1}}^{E_{i_{0}}}$ for some $i_{0} \in I$ with $\left(n_{i_{0}}\right)_{x_{0}} \in l_{0}$. Since $N$ is irreducible, the complex Coxeter group associated with $N$ is not decomposable. Furthermore, since $N$ is full, the group is not decomposed trivially. Hence, according to Lemma 3.8 of [Koi3], we can find a $J$-curvature normal $n_{i_{1}}$ of $N$ satisfying $\left(n_{i_{1}}\right)_{x_{0}} \notin \operatorname{Span}_{\mathbf{C}}\left\{\left(n_{i_{0}}\right)_{x_{0}}\right\} \cup \operatorname{Span}_{\mathbf{C}}\left\{\left(n_{i_{0}}\right)_{x_{0}}\right\}^{\perp}$ (see the final part of the first paragraph of Section 2), where we use also $\operatorname{codim}_{\mathbf{c}} N \geq 2$. Furthermore, since $n_{i_{1}}$ is a $J$-curvature normal, so are also infinitely many complex-constantmultiples of $n_{i_{1}}$. Hence we may assume that $\left(n_{i_{1}}\right)_{x_{0}}$ does not belong to $l_{0}$ by replacing $n_{i_{1}}$ to a complex-constant-multiple of $n_{i_{1}}$ if necessary. Denote by $l_{i_{0} i_{1}}$ the affine line in $T_{x_{0}}^{\perp} N$ through $\left(n_{i_{0}}\right)_{x_{0}}$ and $\left(n_{i_{1}}\right)_{x_{0}}$, and set $D_{i_{0} i_{1}}:=D_{l_{i_{0} i_{1}}}$ for simplicity. According to Theorem 4.4, $L_{x_{j_{0}-1}}^{D_{i_{0} i_{1}}}$ is a principal orbit of the direct sum representation of some aksrepresentations and a trivial representation and hence it is an anti-Kaehler isoparametric submanifold with $J$-diagonalizable shape operators in $\left(W_{l_{i_{0} i_{1}}}\right)_{x_{j_{0}-1}}$ of complex codimension two. Furthermore, since both $\left(n_{i_{0}}\right)_{x_{0}}$ and $\left(n_{i_{1}}\right)_{x_{0}}$ are $J$-curvature normals of $L_{x_{j_{0}-1}}^{D_{i_{0} i_{1}}}\left(\subset\left(W_{l_{i_{0} i_{1}}}\right)_{x_{j_{0}-1}}\right)$ and since they are not orthogonal, it follows from Lemma 3.8 of [Koi3] that $L_{x_{j_{0}-1}}^{D_{i_{0} i_{1}}}$ is irreducible. Hence, by the anti-Kaehler version of Theorem D of [HOT], $x_{j_{0}-1}$ can be joined to $x_{j_{0}}$ by a piecewise smooth curve each of whose smooth segments is tangent to one of $E_{i}$ 's $\left(i \in I\right.$ s.t. $\left(n_{i}\right)_{x_{0}} \in l_{i_{0} i_{1}}$ and $\left.\left(n_{i}\right)_{x_{0}} \neq\left(n_{i_{0}}\right)_{x_{0}}\right)$. Therefore, we can find a sequence $\left\{x_{0}, x_{1}^{\prime}, \cdots, x_{k^{\prime}}^{\prime}(=x)\right\}$ such that, for each $j \in\left\{1, \cdots, k^{\prime}\right\}, x_{j}^{\prime} \in$ $\left(\underset{i \in I \text { s.t. }\left(n_{i}\right)_{x_{0}} \notin l_{0}}{\cup} L_{x_{j-1}^{\prime}}^{E_{i}}\right) \cup L_{x_{j-1}^{\prime}}^{E_{0}}$ holds. Hence it follows from Lemma 4.6 .2 (see below) that $x_{1}^{\prime} \in \widehat{Q}_{0}\left(x_{0}\right), x_{2}^{\prime} \in \widehat{Q}_{0}\left(x_{1}^{\prime}\right), \cdots, x_{k^{\prime}-1}^{\prime} \in \widehat{Q}_{0}\left(x_{k^{\prime}-2}^{\prime}\right)$ and $x \in \widehat{Q}_{0}\left(x_{k^{\prime}-1}^{\prime}\right)$ inductively. Therefore we have $x \in \widehat{Q}_{0}\left(x_{0}\right)$. From the arbitrariness of $x$, it follows that $Q_{0}\left(x_{0}\right) \subset \widehat{Q}_{0}\left(x_{0}\right)$. Similarly we can show $\widehat{Q}_{0}\left(x_{0}\right) \subset Q_{0}\left(x_{0}\right)$. Thus we obtain $Q_{0}\left(x_{0}\right)=\widehat{Q}_{0}\left(x_{0}\right)$ and hence $\overline{Q_{0}\left(x_{0}\right)}=\overline{\widehat{Q}_{0}\left(x_{0}\right)}$. Let $\mathcal{D}_{E}^{0}$ (resp. $\widehat{\mathcal{D}}_{E}^{0}$ ) be the set of all local (smooth) vector fields of $N$ (resp. $\widehat{N}$ ) which is tangent to some $E_{i}$ (resp. $\left.\widehat{E}_{i}\right)(i \in I \cup\{0\})$ at each point of the domain. Since $\overline{\left(\mathcal{D}_{E}^{0}\right)^{*}(x)}=\overline{\left(E_{0}\right)_{x} \oplus\left(\oplus_{i \in I}^{\oplus}\left(E_{i}\right)_{x}\right)}=T_{x} N$ for each $x \in N$, it follows from Theorem 4.1 that $\overline{\Omega_{\mathcal{D}_{E}^{0}}\left(x_{0}\right)}=N$. Similarly, we have $\overline{\Omega_{\widehat{\mathcal{D}}_{E}^{0}}\left(x_{0}\right)}=\widehat{N}$. Also, it is clear that $\Omega_{\mathcal{D}_{E}^{0}}\left(x_{0}\right)=Q_{0}\left(x_{0}\right)$ and $\Omega_{\widehat{\mathcal{D}}_{E}^{0}}\left(x_{0}\right)=\widehat{Q}_{0}\left(x_{0}\right)$. Therefore we obtain $N=\widehat{N} . \quad$ q.e.d. 
Lemma 4.6.2. Let $N, \widehat{N}, x_{0}$ and $l_{0}$ be as in Lemma 4.6.1. Then we have $L_{x}^{D_{l}}=L_{x}^{\widehat{D}_{l}}$ for any $x \in L_{x_{0}}^{E_{0}} \cup\left(\underset{i \in I \text { s.t. }\left(n_{i}\right)_{x_{0}} \notin l_{0}}{\cup} L_{x_{0}}^{E_{i}}\right)$ and any complex affine line $l$ of $T_{x_{0}}^{\perp} N$ with $l \neq l_{0}$. Also, we have $T_{x} N=T_{x} \widehat{N}$ for any $x \in L_{x_{0}}^{E_{0}} \cup\left(\underset{i \in I \text { s.t. }\left(n_{i}\right)_{x_{0}} \notin l_{0}}{\cup} L_{x_{0}}^{E_{i}}\right)$.

Proof. Assume that $x \in L_{x_{0}}^{E_{i_{0}}}$, where $i_{0}$ is an element of $\left\{i \in I \mid\left(n_{i}\right)_{x_{0}} \notin l_{0}\right\} \cup\{0\}$. Take any complex affine line $l$ of $T_{x_{0}}^{\perp} N$ with $l \neq l_{0}$. In case of $\left(n_{i_{0}}\right)_{x_{0}} \in l$, we have $x \in L_{x_{0}}^{E_{i_{0}}} \subset L_{x_{0}}^{D_{l}}=L_{x_{0}}^{\widehat{D}_{l}}$ and hence $L_{x}^{D_{l}}=L_{x}^{\widehat{D}_{l}}$. We consider the case of $\left(n_{i_{0}}\right)_{x_{0}} \notin l$. Take a curve $\gamma:[0,1] \rightarrow L_{x_{0}}^{E_{i_{0}}}$ with $\gamma(0)=x_{0}$ and $\gamma(1)=x$. Since $\left(n_{i_{0}}\right)_{x_{0}} \notin l, \gamma$ is $D_{l}$-horizontal. For the holomorphic isometries $h_{\gamma, 1}^{D_{l}}:\left(W_{l}\right)_{x_{0}} \rightarrow\left(W_{l}\right)_{x}$ and $h_{\gamma, 1}^{\widehat{D}_{l}}:\left(\widehat{W}_{l}\right)_{x_{0}} \rightarrow\left(\widehat{W}_{l}\right)_{x}$ as in Lemma 4.5, we have $h_{\gamma, 1}^{D_{l}}\left(L_{x_{0}}^{D_{l}}\right)=L_{x}^{D_{l}}$ and $h_{\gamma, 1}^{\widehat{D}_{l}}\left(L_{x_{0}}^{\widehat{D}_{l}}\right)=L_{x}^{\widehat{D}_{l}}$. On the other hand, in case of $i_{0} \neq 0$, we can show $h_{\gamma, 1}^{D_{l}}=h_{\gamma, 1}^{\widehat{D}_{l}}$ by imitating the discussion from Line 7 from bottom of Page 164 to Line 4 of Page 165 in [HL2]. Also, in case of $i_{0}=0$, we can show $h_{\gamma, 1}^{D_{l}}=h_{\gamma, 1}^{\widehat{D}_{l}}$ by imitating the discussion from Line 18 of Page 165 to Line 6 of Page 166 in [HL2]. Hence we obtain $L_{x}^{D_{l}}=L_{x}^{\widehat{D}_{l}}$. Therefore we obtain

$$
T_{x} N=\overline{\left(E_{0}\right)_{x} \oplus\left(\oplus_{i \in I}\left(E_{i}\right)_{x}\right)}=\overline{\sum_{l \neq l_{0}} T_{x} L_{x}^{D_{l}}}=\overline{\sum_{l \neq l_{0}} T_{x} L_{x}^{\widehat{D}_{l}}}=\overline{\left(\widehat{E}_{0}\right)_{x} \oplus\left(\underset{i \in \widehat{I}}{\oplus}\left(\widehat{E}_{i}\right)_{x}\right)}=T_{x} \widehat{N} .
$$

This completes the proof.

q.e.d.

In similar to Lemma 4.2 in [HL2], we have the following fact.

Lemma 4.6.3. Let $N$ be a principal orbit of an aks-representation (which is a full irreducible anti-Kaehler isoparametric submanifold with $J$-diagonalizable shape operators). Then each holomorphic isometry of the ambient (finite dimensional) anti-Kaehler space defined for $N$ in similar to the holomorphic isometry $F_{\gamma}$ preserves $N$ invariantly.

Proof. Let $G / K$ be an irreducible anti-Kaehler symmetric space and $(\mathfrak{g}, \tau)$ the anti-Kaehler symmetric Lie algebra associated with $G / K$. Set $\mathfrak{p}:=\operatorname{Ker}(\tau+\mathrm{id})$. Let $\mathfrak{a}_{s}$ be a maximal split abelian subspace of $\mathfrak{p}$ and $\mathfrak{p}=\mathfrak{p}_{0}+\sum_{\alpha \in \triangle_{+}} \mathfrak{p}_{\alpha}$ the root space decomposition with respect to $\mathfrak{a}_{s}$. Set $\mathfrak{a}:=\mathfrak{p}_{0}\left(\supset \mathfrak{a}_{s}\right)$. Let $N$ be the principal orbit of the aks-representation $\rho:=\left.\operatorname{Ad}_{G}\right|_{\mathfrak{p}}: K \rightarrow G L(\mathfrak{p})$ through a regular element $x(\in \mathfrak{a})$. Denote by $A$ the shape tensor of $N$. Take $v \in T_{x}^{\perp} N(=\mathfrak{a})$ and let $\widetilde{v}$ be the parallel normal vector field of $N$ with $\widetilde{v}_{x}=v$. 
Note that $\widetilde{v}_{\rho(k)(x)}=\rho(k)_{* x}(v)$ holds for any $k \in K$. Then we have $T_{x} N=\sum_{\alpha \in \triangle_{+}} \mathfrak{p}_{\alpha}$ and

$$
\left.A_{\widetilde{v}_{\rho(k)(x)}}\right|_{\rho(k)_{* x}\left(\mathfrak{p}_{\alpha}\right)}=-\frac{\alpha^{\mathbf{c}}(v)}{\alpha^{\mathbf{c}}(x)} \mathrm{id}\left(\alpha \in \triangle_{+}\right) .
$$

For each $\alpha \in \triangle_{+}$, define the section $\lambda_{\alpha}$ of the $\mathbf{C}$-dual bundle $\left(T^{\perp} N\right)^{*_{\mathrm{c}}}$ of $T^{\perp} N$ by

$$
\left(\lambda_{\alpha}\right)_{\rho(k)(x)}:=-\frac{\alpha^{\mathbf{c}} \circ \rho(k)_{* x}^{-1}}{\alpha^{\mathbf{c}}(x)} \quad(k \in K)
$$

Since $\rho(k)_{* x}$ is the parallel translation along any curve $c$ in $N$ connecting $x$ and $\rho(k)(x)$ with respect to the normal connection of $N, \lambda_{\alpha}$ is a parallel section of $\left(T^{\perp} N\right)^{*_{\mathrm{c}}}$. It follows from (4.1) that $\left\{\lambda_{\alpha} \mid \alpha \in \triangle_{+}\right\}$is the set of all $J$-principal curvatures of $N$. Let $E_{\alpha}$ be the $J$-curvature distribution for $\lambda_{\alpha}$. Take $\alpha_{0} \in \triangle_{+}$and $v_{0} \in\left(\lambda_{\alpha_{0}}\right)_{x}^{-1}(1) \backslash$ $\left(\cup_{\alpha \in \triangle_{+} \text {s.t. } \alpha \neq \alpha_{0}}\left(\lambda_{\alpha}\right)_{x}^{-1}(1)\right)$ and set $F:=\rho(K) \cdot\left(x+v_{0}\right)$. It is clear that $F$ is a focal submanifold of $N$ whose corresponding focal distribution is equal to $E_{\alpha_{0}}$. Denote by $K_{x}$ (resp. $\left.K_{x+v_{0}}\right)$ the isotropy group of the $\rho(K)$-action at $x$ (resp. $\left.x+v_{0}\right)$ and $\mathfrak{k}_{x}\left(\right.$ resp. $\left.\mathfrak{k}_{x+v_{0}}\right)$ the Lie algebra of $K_{x}$ (resp. $\left.K_{x+v_{0}}\right)$. The restriction of the $\rho\left(K_{x+v_{0}}\right)$-action to $T_{x+v_{0}}^{\perp} F$ is called the slice representation of the $\rho(K)$-action at $x+v_{0}$. It is shown that this slice representation coincides with the normal holonomy group action of $F$ at $x+v_{0}$ and that $\rho\left(K_{x+v_{0}}\right) \cdot x$ is equal to $L_{x}^{E_{\alpha_{0}}}$. Set $\Psi\left(x+v_{0}\right):=\rho\left(K_{x+v_{0}}\right)$ and $\Psi(x):=\rho\left(K_{x}\right)$. The leaf $L_{x}^{E_{\alpha_{0}}}$ is identified with the quotient manifold $\Psi\left(x+v_{0}\right) / \Psi(x)$. Take $X\left(=\operatorname{ad}_{\mathfrak{g}}(\bar{X})\right) \in \operatorname{Lie} \Psi\left(x+v_{0}\right) \ominus \operatorname{Lie} \Psi(x)$, where $\bar{X} \in \mathfrak{k}_{x+v_{0}}$, and set $g(t):=\exp _{\Psi\left(x+v_{0}\right)}(t X)$ and $\gamma(t):=g(t) \cdot x$, where $t \in[0,1]$. Let $F_{\gamma}$ be the holomorphic isometry of the ambient anti-Kaehler space satisfying $F_{\gamma}(x)=$ $\gamma(1),\left.\left(F_{\gamma}\right)_{* x}\right|_{\left(E_{\alpha_{0}}\right)_{x}}=\left.g(1)_{* x}\right|_{\left(E_{\alpha_{0}}\right)_{x}},\left.\left(F_{\gamma}\right)_{* x}\right|_{\left(E_{\alpha}\right)_{x}}=\left.h_{\gamma, 1}^{E_{\alpha}}\right|_{\left(E_{\alpha}\right)_{x}}\left(\alpha \in \triangle_{+}\right.$s.t. $\left.\alpha \neq \alpha_{0}\right)$ and $\left.\left(F_{\gamma}\right)_{* x}\right|_{T_{x}^{\perp} N}=\tau_{\gamma}^{\perp}$, where $h_{\gamma, 1}^{E_{\alpha}}$ is the holomorphic isometry defined in similar to $h_{\gamma, t}^{D_{P}}$ in the statement of Lemma 4.5 and $\tau_{\gamma}^{\perp}$ is the parallel translation along $\gamma$ with respect to the normal connection of $N$. Easily we can show $\left.h_{\gamma, 1}^{E_{\alpha}}\right|_{\left(E_{\alpha}\right)_{x}}=\left.g(1)_{* x}\right|_{\left(E_{\alpha}\right)_{x}}$ and $\tau_{\gamma}^{\perp}=\left.g(1)_{* x}\right|_{T_{x}^{\perp} N}$. Hence we have $\left(F_{\gamma}\right)_{* x}=g(1)_{* x}$. Furthermore, since both $F_{\gamma}$ and $g(1)$ are affine transformations of the ambient anti-Kaehler space, they coincide with each other. Therefore, we obtain $F_{\gamma}(N)=g(1)(\rho(K) \cdot x)=\rho\left(\exp _{G}(\bar{X})\right)(\rho(K) \cdot x)=N$. This completes the proof.

q.e.d.

By using Lemmas 4.6.1 and 4.6.3, we shall prove Proposition 4.6.

Proof of Proposition 4.6. Since $M$ is a full irreducible anti-Kaehler isoparametric submanifold with $J$-diagonalizable shape operators and $F_{\gamma}$ is a holomorphic isometry of $V$, $\widehat{M}:=F_{\gamma}(M)$ also is a full irreducible anti-Kaehler isoparametric one with $J$-diagonalizable shape operators. Denote by $\widehat{A}$ the shape tensor of $\widehat{M}$. Let $\left\{\widehat{E}_{0}\right\} \cup\left\{\widehat{E}_{i} \mid i \in \widehat{I}\right\}$ be the 
set of all $J$-curvature distributions on $\widehat{M}$ and $\widehat{n}_{i}$ the $J$-curvature normal corresponding to $\widehat{E}_{i}$, where $\widehat{E}_{0}$ is a distribution on $\widehat{M}$ defined by $\left(\widehat{E}_{0}\right)_{x}:=\bigcap_{v \in T_{x}^{\perp} \widehat{M}} \operatorname{Ker} \widehat{A}_{v}(x \in \widehat{M})$. Clearly we may assume that $\widehat{I}=I$ and $\widehat{E}_{i}=\left(F_{\gamma}\right)_{*}\left(E_{i}\right)(i \in I \cup\{0\})$. Also we have $\gamma(1) \in M \cap \widehat{M}$. Since $\left(F_{\gamma}\right)_{* \gamma(0)}\left(\left(n_{i}\right)_{\gamma(0)}\right)=\tau_{\gamma}^{\perp}\left(\left(n_{i}\right)_{\gamma(0)}\right)=\left(n_{i}\right)_{\gamma(1)}(i \in I)$, we have $\left(\widehat{n}_{i}\right)_{\gamma(1)}=\left(n_{i}\right)_{\gamma(1)}(i \in I \cup\{0\})$. Also, since $\left(F_{\gamma}\right)_{* \gamma(0)}\left(\left(E_{i}\right)_{\gamma(0)}\right)=\left(h_{\gamma, 1}^{E_{i}}\right)_{* \gamma(0)}\left(\left(E_{i}\right)_{\gamma(0)}\right)=$ $\left(E_{i}\right)_{\gamma(1)}\left(i \in(I \cup\{0\}) \backslash\left\{i_{0}\right\}\right)$, we have $\left(\widehat{E}_{i}\right)_{\gamma(1)}=\left(E_{i}\right)_{\gamma(1)}\left(i \in(I \cup\{0\}) \backslash\left\{i_{0}\right\}\right)$. Also, since $\left(F_{\gamma}\right)_{* \gamma(0)}\left(\left(E_{i_{0}}\right)_{\gamma(0)}\right)=g(1)_{* \gamma(0)}\left(\left(E_{i_{0}}\right)_{\gamma(0)}\right)=\left(E_{i_{0}}\right)_{\gamma(1)}$, we have $\left(\widehat{E}_{i_{0}}\right)_{\gamma(1)}=\left(E_{i_{0}}\right)_{\gamma(1)}$. From these facts, we have $L_{\gamma(1)}^{\widehat{E}_{i}}=L_{\gamma(1)}^{E_{i}}(i \in I \cup\{0\})$ and $T_{\gamma(1)} M=T_{\gamma(1)} \widehat{M}$. Let $l_{0}$ be the complex affine line through 0 and $\left(n_{i_{0}}\right)_{\gamma(1)}$. Take any complex affine line $l$ of $T_{\gamma(1)}^{\perp} M$ with $l \neq l_{0}$. Now we shall show that $L_{\gamma(1)}^{D_{l}}=L_{\gamma(1)}^{\widehat{D}_{l}}$, where $D_{l}$ (resp. $\widehat{D}_{l}$ ) is the distribution on $M$ (resp. $\widehat{M}$ ) defined as above for $l$. If $\left(n_{i_{0}}\right)_{\gamma(1)} \notin l$, then $\gamma$ is a $D_{l}$-horizontal curve and hence we have $F_{\gamma}\left(L_{x_{0}}^{D_{l}}\right)=h_{\gamma, 1}^{D_{l}}\left(L_{x_{0}}^{D_{l}}\right)=L_{\gamma(1)}^{D_{l}}$ and hence $L_{\gamma(1)}^{\widehat{D}_{l}}=L_{\gamma(1)}^{D_{l}}$. Next we consider the case of $\left(n_{i_{0}}\right)_{\gamma(1)} \in l$. Then we have $0 \notin l$. If there does not exist $i_{1}\left(\neq i_{0}\right) \in I$ with $\left(n_{i_{1}}\right)_{\gamma(1)} \in l$, then we have $L_{\gamma(1)}^{D_{l}}=L_{\gamma(1)}^{E_{i_{0}}}=L_{\gamma(1)}^{\widehat{E}_{i_{0}}}=L_{\gamma(1)}^{\widehat{D}_{l}}$. Next we consider the case where there exists $i_{1}\left(\neq i_{0}\right) \in I$ with $\left(n_{i_{1}}\right)_{\gamma(1)} \in l$. Let $\widetilde{v}$ be a focal normal vector field of $M$ such that the corresponding focal distribution is equal to $D_{l}$. Since $0 \notin l$, it follows from Theorem 4.4 that $L_{\gamma(1)}^{D_{l}}$ is a principal orbit of the direct sum representation of aks-representations and a trivial representation. Since $\left(n_{i_{0}}\right)_{\gamma(1)},\left(n_{i_{1}}\right)_{\gamma(1)} \in l$ and $0 \notin l,\left(n_{i_{0}}\right)_{\gamma(1)}$ and $\left(n_{i_{1}}\right)_{\gamma(1)}$ are C-linearly independent. Assume that $L_{\gamma(1)}^{D_{l}}$ is reducible. Then the complex Coxeter group associated with $L_{\gamma(1)}^{D_{l}}$ is decomposable. Hence $\left(n_{i_{0}}\right)_{\gamma(1)}$ and $\left(n_{i_{1}}\right)_{\gamma(1)}$ are orthogonal and there exists no $J$-curvature normal of $L_{\gamma(1)}^{D_{l}}$ other than them. Therefore, $L_{\gamma(1)}^{D_{l}}$ is congruent to the (extrinsic) product of complex spheres $L_{\gamma(1)}^{E_{i_{0}}}$ and $L_{\gamma(1)}^{E_{i_{1}}}$. Similarly $L_{x_{0}}^{D_{l}}$ is congruent to the (extrinsic) product of $L_{x_{0}}^{E_{i_{0}}}$ and $L_{x_{0}}^{E_{i_{1}}}$. Therefore we have

$$
F_{\gamma}\left(L_{x_{0}}^{D_{l}}\right)=F_{\gamma}\left(L_{x_{0}}^{E_{i_{0}}}\right) \times F_{\gamma}\left(L_{x_{0}}^{E_{i_{1}}}\right)=L_{\gamma(1)}^{E_{i_{0}}} \times L_{\gamma(1)}^{E_{i_{1}}}=L_{\gamma(1)}^{D_{l}}
$$

and hence $L_{\gamma(1)}^{\widehat{D}_{l}}=L_{\gamma(1)}^{D_{l}}$. Assume that $L_{\gamma(1)}^{D_{l}}$ is irreducible. Then $L_{\gamma(1)}^{D_{l}}$ is a principal orbit of an aks-representation. Then it follows from Lemma 4.6.3 that $F_{\gamma}\left(L_{x_{0}}^{D_{l}}\right)=$ $\left(\left.F_{\gamma}\right|_{\left(W_{l}\right)_{x_{0}}}\right)\left(L_{x_{0}}^{D_{l}}\right)=L_{x_{0}}^{D_{l}}$. Hence we obtain $L_{\gamma(1)}^{\widehat{D}_{l}}=L_{\gamma(1)}^{D_{l}}$. Thus we obtain $L_{\gamma(1)}^{\widehat{D}_{l}}=L_{\gamma(1)}^{D_{l}}$ in general. Therefore, from Lemma 4.6.1, we obtain $M=\widehat{M}=F_{\gamma}(M)$, that is, $F_{\gamma}(M)=M$.

q.e.d.

By using Proposition 4.6, we prove the following fact. 
Proposition 4.7. For any $x \in Q\left(x_{0}\right)$, there exists a holomorphic isometry $f$ of $V$ such that $f\left(x_{0}\right)=x, f(M)=M, f_{*}\left(E_{i}\right)=E_{i}(i \in I), f\left(Q\left(x_{0}\right)\right)=Q\left(x_{0}\right)$ and that $\left.f_{* x_{0}}\right|_{T_{x_{0}}^{\perp} M}$ coincides with the parallel translation along a curve in $M$ connecting $x_{0}$ and $x$ with respect to the normal connection of $M$.

Proof. Take a sequence $\left\{x_{0}, x_{1}, \cdots, x_{k}(=x)\right\}$ of $Q\left(x_{0}\right)$ such that, for each $i \in\{0,1, \cdots, k-$ $1\}, x_{i}$ and $x_{i+1}$ belong to a complex curvature sphere $S_{i}^{\mathbf{c}}$ of $M$. Furthermore, for each $i \in\{0,1, \cdots, k-1\}$, we take the geodesic $\gamma_{i}:[0,1] \rightarrow S_{i}^{\mathbf{c}}$ with $\gamma_{i}(0)=x_{i}$ and $\gamma_{i}(1)=x_{i+1}$. Set $f:=F_{\gamma_{k-1}} \circ \cdots \circ F_{\gamma_{1}} \circ F_{\gamma_{0}}$, where $F_{\gamma_{i}}(i=0,1, \cdots, k-1)$ are holomorphic isometries of $V$ defined in similar to the above $F_{\gamma}$. According to Proposition 4.6, $f$ preserves $M$ invariantly, $f_{*}\left(E_{i}\right)=E_{i}(i \in I)$ and the restriction of $f_{* x_{0}}$ to $T_{x_{0}}^{\perp} M$ coincides with the parallel translation along a curve in $M$ connecting $x_{0}$ and $x$ with respect to the normal connection of $M$. Also, since $f$ preserves complex curvature spheres invariantly, it is shown that $f$ preserves $Q\left(x_{0}\right)$ invariantly. Thus $f$ is the desired holomorphic isometry.

q.e.d.

By using Propositions 4.2 and 4.7, we shall prove Theorem A.

Proof of Theorem A. Take any $\widehat{x} \in M$. Since $\overline{Q\left(x_{0}\right)}=M$ by Proposition 4.2 , there exists a sequence $\left\{x_{k}\right\}_{k=1}^{\infty}$ in $Q\left(x_{0}\right)$ with $\lim _{k \rightarrow \infty} x_{k}=\widehat{x}$. According to Proposition 4.7, for each $k \in \mathbf{N}$, there exists a holomorphic isometry $f_{k}$ of $V$ with $f_{k}\left(x_{0}\right)=x_{k}, f_{k}(M)=$ $M, f_{k}\left(Q\left(x_{0}\right)\right)=Q\left(x_{0}\right)$ and $f_{k}\left(L_{x_{0}}^{E_{i}}\right)=L_{x_{k}}^{E_{i}}(i \in I)$.

(Step I) In this step, we shall show that, for each $i \in I$, there exists a subsequence $\left\{f_{k_{j}}\right\}_{j=1}^{\infty}$ of $\left\{f_{k}\right\}_{k=1}^{\infty}$ such that $\left\{\left.f_{k_{j}}\right|_{\left.L_{x_{0}}\right\}_{i}}\right\}_{j=1}^{\infty}$ pointwisely converges to a holomorphic isometry of $L_{x_{0}}^{E_{i}}$ onto $L_{\widehat{x}}^{E_{i}}$. For any point $x$ of $M$, denote by $\left(L_{x}^{E_{i}}\right)_{\mathbf{R}}$ the compact real form through $x$ of the complex sphere $L_{x}^{E_{i}}$ satisfying $\left\langle T_{x}\left(L_{x}^{E_{i}}\right)_{\mathbf{R}}, J T_{x}\left(L_{x}^{E_{i}}\right)_{\mathbf{R}}\right\rangle=0$, where a real form of $L_{x}^{E_{i}}$ means the fixed point set of an anti-holomorphic diffeomorphism of $L_{x}^{E_{i}}$. Note that such a compact real form $\left(L_{x}^{E_{i}}\right)_{\mathbf{R}}$ of $L_{x}^{E_{i}}$ is determined uniquely (see Figure 3 ) and that it is isometric to a $m_{i}$-dimensional sphere, where $m_{i}:=\operatorname{dim}_{\mathbf{c}} E_{i}$. Clearly we have $f_{k}\left(\left(L_{x_{0}}^{E_{i}}\right)_{\mathbf{R}}\right)=\left(L_{x_{k}}^{E_{i}}\right)_{\mathbf{R}}$. Denote by $\mathfrak{F}_{i}$ the foliation on $M$ whose leaf through $x \in M$ is equal to $\left(L_{x}^{E_{i}}\right)_{\mathbf{R}}$. Take a $\mathfrak{F}_{i^{-}}$-saturated tubular neighborhood $U$ of $\left(L_{\widehat{x}}^{E_{i}}\right)_{\mathbf{R}}$ in $M$, where " $\mathfrak{F}_{i}$-saturatedness" of $U$ means that $\left(L_{x}^{E_{i}}\right)_{\mathbf{R}} \subset U$ for any $x \in U$. Take a base $\left\{e_{1}, \cdots, e_{m_{i}}\right\}$ of $T_{x_{0}}\left(\left(L_{x_{0}}^{E_{i}}\right)_{\mathbf{R}}\right)$ such that the norms $\left\|e_{1}\right\|, \cdots,\left\|e_{m_{i}}\right\|$ are sufficiently small and set $\bar{x}_{a}:=\exp _{x_{0}}\left(e_{a}\right)\left(a=1, \cdots, m_{i}\right)$, where $\exp _{x_{0}}$ is the exponential map of $\left(L_{x_{0}}^{E_{i}}\right)_{\mathbf{R}}$ at $x_{0}$. Since $\left(L_{x}^{E_{i}}\right)_{\mathbf{R}}$ 's $(x \in U)$ are compact, $\mathfrak{F}_{i}$ is a Hausdorff foliation. From this fact and the compactness of $\left(L_{\widehat{x}}^{E_{i}}\right)_{\mathbf{R}}$, it follows that there exists a subsequence $\left\{f_{k_{j}}\right\}_{j=1}^{\infty}$ of $\left\{f_{k}\right\}_{k=1}^{\infty}$ such that $\left\{f_{k_{j}}\left(x_{0}\right)\right\}_{j=1}^{\infty}$ and $\left\{f_{k_{j}}\left(\bar{x}_{a}\right)\right\}_{j=1}^{\infty}\left(a=1, \cdots, m_{i}\right)$ converge. Set $\widehat{x}:=\lim _{j \rightarrow \infty} f_{k_{j}}\left(x_{0}\right)$ and 
$\widehat{x}_{a}:=\lim _{j \rightarrow \infty} f_{k_{j}}\left(\bar{x}_{a}\right)\left(a=1, \cdots, m_{i}\right)$. Since $\lim _{j \rightarrow \infty} f_{k_{j}}\left(x_{0}\right)=\widehat{x}$ and $f_{k_{j}}\left(\left(L_{x_{0}}^{E_{i}}\right)_{\mathbf{R}}\right)=\left(L_{x_{k_{j}}}^{E_{i}}\right)_{\mathbf{R}}$, it follows from the Hausdorffness of $\mathfrak{F}_{i}$ that $\widehat{x}_{a}$ belongs to $\left(L_{\widehat{x}}^{E_{i}}\right)_{\mathbf{R}}\left(a=1, \cdots, m_{i}\right)$. Denote by $d_{0}, d_{j}(j \in \mathbf{N})$ and $\widehat{d}$ the (Riemannian) distance functions of $\left(L_{x_{0}}^{E_{i}}\right)_{\mathbf{R}},\left(L_{x_{k_{j}}}^{E_{i}}\right)_{\mathbf{R}}$ and $\left(L_{\widehat{x}}^{E_{i}}\right)_{\mathbf{R}}$, respectively. Since each $\left.f_{k_{j}}\right|_{\left(L_{x_{0}}^{E_{i}}\right)_{\mathbf{R}}}$ is an isometry onto $\left(L_{x_{k_{j}}}^{E_{i}}\right)_{\mathbf{R}}$, we have $d_{j}\left(f_{k_{j}}\left(x_{0}\right), f_{k_{j}}\left(\bar{x}_{a}\right)\right)=d_{0}\left(x_{0}, \bar{x}_{a}\right)$ and $d_{j}\left(f_{k_{j}}\left(\bar{x}_{a}\right), f_{k_{j}}\left(\bar{x}_{b}\right)\right)=d_{0}\left(\bar{x}_{a}, \bar{x}_{b}\right),\left(a, b=1, \cdots, m_{i}\right)$. Hence we have $\widehat{d}\left(\widehat{x}, \widehat{x}_{a}\right)=d_{0}\left(x_{0}, \bar{x}_{a}\right)$ and $\widehat{d}\left(\widehat{x}_{a}, \widehat{x}_{b}\right)=d_{0}\left(\bar{x}_{a}, \bar{x}_{b}\right)\left(a, b=1, \cdots, m_{i}\right)$. Therefore, since $\left(L_{x_{0}}^{E_{i}}\right)_{\mathbf{R}}$ and $\left(L_{\widehat{x}}^{E_{i}}\right)_{\mathbf{R}}$ are spheres isometric to each other, there exists a unique isometry $\bar{f}$ of $\left(L_{x_{0}}^{E_{i}}\right)_{\mathbf{R}}$ onto $\left(L_{\widehat{x}}^{E_{i}}\right)_{\mathbf{R}}$ satisfying $\bar{f}\left(x_{0}\right)=\widehat{x}$ and $\bar{f}\left(\bar{x}_{a}\right)=\widehat{x}_{a}\left(a=1, \cdots, m_{i}\right)$. It is clear that $\bar{f}$ is uniquely extended to a holomorphic isometry of $L_{x_{0}}^{E_{i}}$ onto $L_{\widehat{x}}^{E_{i}}$. Denote by $f$ this holomorphic extension. It is easy to show that $\left\{\left.f_{k_{j}}\right|_{\left(L_{x_{0}}^{E_{i}}\right)_{\mathbf{R}}}\right\}_{j=1}^{\infty}$ pointwisely converges to $\bar{f}$. Furthermore, it follows from this fact that $\left\{\left.f_{k_{j}}\right|_{L_{x_{0}} E_{i}}\right\}_{j=1}^{\infty}$ pointwisely converges to $f$.

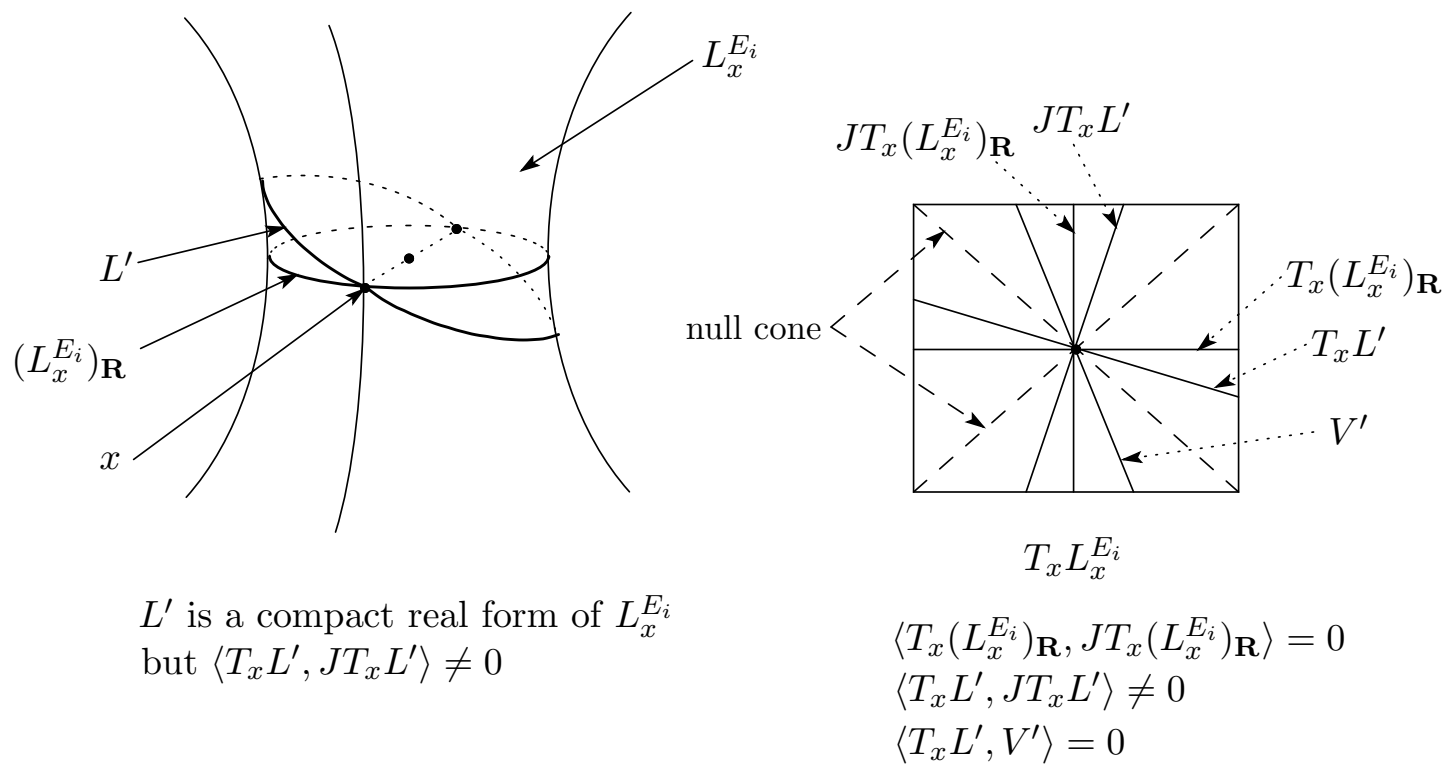

Figure 3.

(Step II) Next we shall show that, for each fixed $y \in Q\left(x_{0}\right)$, there exists a subsequence $\left\{f_{k_{j}}\right\}_{j=1}^{\infty}$ of $\left\{f_{k}\right\}_{k=1}^{\infty}$ such that $\left\{f_{k_{j}}(y)\right\}_{j=1}^{\infty}$ converges. There exists a sequence $\left\{\bar{x}_{0}\left(=x_{0}\right), \bar{x}_{1}, \cdots, \bar{x}_{m}(=y)\right\}$ in $Q\left(x_{0}\right)$ such that, for each $j \in\{1, \cdots, m\}, \bar{x}_{j}$ is contained in a complex curvature sphere through $\bar{x}_{j-1}$ (which we denote by $L_{\bar{x}_{j-1}}^{E_{i(j)}}$ ). For simplicity, we shall consider the case of $m=2$. From the fact in Step I, there exists a subsequence 
$\left\{f_{k_{j}^{1}}\right\}_{j=1}^{\infty}$ of $\left\{f_{k}\right\}_{k=1}^{\infty}$ such that $\left\{\left.f_{k_{j}^{1}}\right|_{L_{x_{0}}} ^{E_{i(1)}}\right\}_{j=1}^{\infty}$ pointwisely converges to a holomorphic isometry $f^{1}$ of $L_{x_{0}}^{E_{i(1)}}$ onto $L_{\widehat{x}}^{E_{i(1)}}$. Furthermore, by noticing $\lim _{j \rightarrow \infty} f_{k_{j}^{1}}\left(\bar{x}_{1}\right)=f^{1}\left(\bar{x}_{1}\right)$ and imitating the discussion in Step I, we can show that there exists a subsequence $\left\{f_{k_{j}^{2}}\right\}_{j=1}^{\infty}$ of $\left\{f_{k_{j}^{1}}\right\}_{j=1}^{\infty}$ such that $\left\{\left.f_{k_{j}^{2}}\right|_{L_{\bar{x}_{1}}^{E_{i(2)}}}\right\}_{j=1}^{\infty}$ pointwisely converges to a holomorphic isometry $f^{2}$ of $L_{\bar{x}_{1}}^{E_{i(2)}}$ onto $L_{f^{1}\left(\bar{x}_{1}\right)}^{E_{i(2)}}$. Since $y=\bar{x}_{2} \in L_{\bar{x}_{1}}^{E_{i(2)}}$, we have $\lim _{j \rightarrow \infty} f_{k_{j}^{2}}(y)=f^{2}(y)$. Thus $\left\{f_{k_{j}^{2}}\right\}_{j=1}^{\infty}$ is the desired subsequence of $\left\{f_{k_{j}}\right\}_{j=1}^{\infty}$.

(Step III) Let $W$ be the complex affine span of $M$. Next we shall show that there exists a subsequence $\left\{f_{k_{j}}\right\}_{j=1}^{\infty}$ of $\left\{f_{k}\right\}_{k=1}^{\infty}$ such that $\left\{\left.f_{k_{j}}\right|_{W}\right\}_{j=1}^{\infty}$ pointwisely converges to some holomorphic isometry of $W$. Take a countable subset $B:=\left\{w_{j} \mid j \in \mathbf{N}\right\}$ of $Q\left(x_{0}\right)$ with $\bar{B}=\overline{Q\left(x_{0}\right)}(=M)$. According to the fact in Step II, there exists a subsequence $\left\{f_{k_{j}^{1}}\right\}_{j=1}^{\infty}$ of $\left\{f_{k}\right\}_{k=1}^{\infty}$ such that $\left\{f_{k_{j}^{1}}\left(w_{1}\right)\right\}_{j=1}^{\infty}$ converges. Again, according to the fact in Step II, there exists a subsequence $\left\{f_{k_{j}^{2}}\right\}_{j=1}^{\infty}$ of $\left\{f_{k_{j}^{1}}\right\}_{j=1}^{\infty}$ such that $\left\{f_{k_{j}^{2}}\left(w_{2}\right)\right\}_{j=1}^{\infty}$ converges. In the sequel, we take subsequences $\left\{f_{k_{j}^{l}}\right\}_{j=1}^{\infty}(l=3,4,5, \cdots)$ inductively. It is clear that $\left\{f_{k_{j}^{j}}\left(w_{l}\right)\right\}_{j=1}^{\infty}$ converges for each $l \in \mathbf{N}$, that is, $\left\{\left.f_{k_{j}^{j}}\right|_{B}\right\}_{j=1}^{\infty}$ pointwisely converges to some map $f$ of $B$ into $M$. Since each $f_{k_{j}^{j}}$ is a holomorphic isometry and hence $\left.f_{k_{j}^{j}}\right|_{W}: W \rightarrow W$ is an affine transformation, $f$ extends to an affine transformation of $W$. Denote by $\tilde{f}$ this extension. It is clear that $\left\{\left.f_{k_{j}^{j}}\right|_{W}\right\}_{j=1}^{\infty}$ pointwisely converges to $\widetilde{f}$ and that $\widetilde{f}$ is a holomorphic isometry of $W$.

(Step IV) Denote by $H$ the group generated by all holomorphic isometries of $V$ preserving $M$ invariantly. Let $\widetilde{f}$ be as in Step III. It is clear that $\widetilde{f}$ extends to a holomorphic isometry of $V$. Denote by $\widehat{f}$ this extension. It is clear that $\widehat{f}(M)=M$ and $\widehat{f}\left(x_{0}\right)=\lim _{j \rightarrow \infty} f_{k_{j}^{j}}\left(x_{0}\right)=\lim _{j \rightarrow \infty} x_{k_{j}^{j}}=\widehat{x}$. Hence we have $\widehat{x} \in H \cdot x_{0}$. From the arbitrariness of $\widehat{x}$, we obtain $M \subset H \cdot x_{0}$. On the ther hand, it is clear that $H \cdot x_{0} \subset M$. Therefore we obtain $H \cdot x_{0}=M$.

q.e.d.

\section{References}

[Be] M. Berger, Les espaces symétriques non compacts, Ann. Sci. Éc. Norm. Supér. III. Sér. 74 (1959) 85-177.

[Br] M. Brück, Equifocal famlies in symmetric spaces of compact type, J.reine angew. Math. 515 (1999), 73-95.

[BCO] J. Berndt, S. Console and C. Olmos, Submanifolds and holonomy, Research Notes in Mathematics 434, CHAPMAN \& HALL/CRC Press, Boca Raton, London, New York Washington, 2003. 
[BH] R. A. Blumenthal and J. J. Hebda, Complementary distributions which preserves the leaf geometry and applications to totally geodesic foliations, Quat. J. Math. 35 (1984) 383-392.

[Ca] E. Cartan, Familles de surfaces isoparamétriques dans les espaces á courbure constante, Ann. Mat. Pura Appl. 17 (1938), 177-191.

[CP] M. Cahen and M. Parker, Pseudo-riemannian symmetric spaces, Memoirs of the Amer. Math. Soc. 24 No. 229 (1980).

[Ch] U. Christ, Homogeneity of equifocal submanifolds, J. Differential Geom. 62 (2002), 1-15.

[Cox] H. S. M. Coxeter, Discrete groups generated by reflections, Ann. of Math. (2) 35 (1934), 588-621.

[E] H. Ewert, Equifocal submanifolds in Riemannian symmetric spaces, Doctoral thesis.

[G1] L. Geatti, Invariant domains in the complexfication of a noncompact Riemannian symmetric space, J. Algebra 251 (2002), 619-685.

[G2] L. Geatti, Complex extensions of semisimple symmetric spaces, manuscripta math. 120 (2006) $1-25$.

[GG] L. Geatti and C. Gorodski, Polar orthogonal representations of real reductive algebraic groups, J. Algebra 320 (2008) 3036-3061.

[Ha] J. Hahn, Isotropy representations of semisimple symmetric spaces and homogeneous hypersurfaces, J. Math. Soc. Japan 40 (1988) 271-288.

[HL1] E. Heintze and X. Liu, A splitting theorem for isoparametric submanifolds in Hilbert space, J. Differential Geom. 45 (1997), 319-335.

[HL2] E. Heintze and X. Liu, Homogeneity of infinite dimensional isoparametric submanifolds, Ann. of Math. 149 (1999), 149-181.

[HLO] E. Heintze, X. Liu and C. Olmos, Isoparametric submanifolds and a Chevalley type restricction theorem, Integrable systems, geometry, and topology, 151-190, AMS/IP Stud. Adv. Math. 36, Amer. Math. Soc., Providence, RI, 2006.

[HOT] E. Heintze, C. Olmos and G. Thorbergsson, Submanifolds with constant prinicipal curvatures and normal holonomy groups, Int. J. Math. 2 (1991), 167-175.

[HPTT] E. Heintze, R. S. Palais, C. L. Terng and G. Thorbergsson, Hyperpolar actions on symmetric spaces, Geometry, topology and physics, 214-245 Conf. Proc. Lecture Notes Geom. Topology 4, Internat. Press, Cambridge, Mass., 1995.

[He] S. Helgason, Differential geometry, Lie groups and symmetric spaces, Pure Appl. Math. 80, Academic Press, New York, 1978.

[Hu] M. C. Hughes, Complex reflection groups, Comm. Algebra 18 (1990), 3999-4029.

[KN] S. Kobayashi and K. Nomizu, Foundations of differential geometry, Interscience Tracts in Pure and Applied Mathematics 15, Vol. II, New York, 1969.

[Koi1] N. Koike, Submanifold geometries in a symmetric space of non-compact type and a pseudoHilbert space, Kyushu J. Math. 58 (2004), 167-202.

[Koi2] N. Koike, Complex equifocal submanifolds and infinite dimensional anti-Keahlerian isoparametric submanifolds, Tokyo J. Math. 28 (2005), 201-247.

[Koi3] N. Koike, A splitting theorem for proper complex equifocal submanifolds, Tohoku Math. J. 58 (2006) 393-417.

[Koi4] N. Koike, The homogeneous slice theorem for the complete complexification of a proper complex equifocal submanifold, Tokyo J. Math. 33 (2010), 1-30.

[Koi5] N. Koike, Hermann type actions on a pseudo-Riemannian symmetric space, Tsukuba J. Math. 34 (2010), 137-172. 
[O] C. Olmos, Isoparametric submanifolds and their homogeneous structures, J. Differential Geom. 38 (1993), 225-234.

[OW] C. Olmos and A. Will, Normal holonomy in Lorentzian space and submanifold geometry, Indiana Univ. Math. J. 50 (2001), 1777-1788.

[O'N] B. O'Neill, Semi-Riemannian Geometry, with applications to relativity, Pure Appl. Math. 103, Academic Press, New York, 1983.

[OS] T. Ohshima and J. Sekiguchi, The restricted root system of a semisimple symmetric pair, Group representations and systems of differential equations (Tokyo, 1982), 433-497, Adv. Stud. Pure Math. 4, North-Holland, Amsterdam, 1984.

[Pa] R. S. Palais, Morse theory on Hilbert manifolds, Topology 2 (1963), 299-340.

[PT2] R. S. Palais and C. L. Terng, Critical point theory and submanifold geometry, Lecture Notes in Math. 1353, Springer-Verlag, Berlin, 1988.

[Pe] A. Z. Petrov, Einstein spaces, Pergamon Press, 1969.

[R] W. Rossmann, The structures of semisimple symmetric spaces, Canad. J. Math. 31 (1979), $157-180$.

[Si] J. Simons, On the transitivity of holonomy systems, Ann. of Math. 76 (1962), 213-234.

[Sz1] R. Szöke, Involutive structures on the tangent bundle of symmetric spaces, Math. Ann. 319 (2001), 319-348.

[Sz2] R. Szöke, Canonical complex structures associated to connections and complexifications of Lie groups, Math. Ann. 329 (2004), 553-591.

[Te1] C. L. Terng, Isoparametric submanifolds and their Coxeter groups, J. Differential Geom. 21 (1985), 79-107.

[Te2] C. L. Terng, Proper Fredholm submanifolds of Hilbert space, J. Differential Geom. 29 (1989), 9-47.

[Te3] C. L. Terng, Polar actions on Hilbert space, J. Geom. Anal. 5 (1995), 129-150.

[TT] C. L. Terng and G. Thorbergsson, Submanifold geometry in symmetric spaces, J. Differential Geom. 42 (1995), 665-718.

[Th] G. Thorbergsson, Isoparametric foliations and their buildings, Ann of Math. 133 (1991), 429-446.

[W1] H. Wu, Holonomy groups of indefinite metrics, Pacific J. Math. 20 (1967), 351-392.

[W2] B. Wu, Isoparametric submanifolds of hyperbolic spaces, Trans. Amer. Math. Soc. 331 (1992), 609-626. 